Valentino Magnani

\title{
Characteristic points, rectifiability and perimeter measure on stratified groups
}

Received November 11, 2004 and in revised form June 10, 2005

\begin{abstract}
We establish an explicit connection between the perimeter measure of an open set $E$ with $C^{1}$ boundary and the spherical Hausdorff measure $\mathcal{S}^{Q-1}$ restricted to $\partial E$, when the ambient space is a stratified group endowed with a left invariant sub-Riemannian metric and $Q$ denotes the Hausdorff dimension of the group. Our formula implies that the perimeter measure of $E$ is less than or equal to $\mathcal{S}^{Q-1}(\partial E)$ up to a dimensional factor. The validity of this estimate positively answers a conjecture raised by Danielli, Garofalo and Nhieu. The crucial ingredient of this result is the negligibility of "characteristic points" of the boundary. We introduce the notion of "horizontal point", which extends the notion of characteristic point to arbitrary submanifolds, and we prove that the set of horizontal points of a $k$-codimensional submanifold is $\mathcal{S}^{Q-k}$-negligible. We propose an intrinsic notion of rectifiability for subsets of higher codimension, called $\left(\mathbb{G}, \mathbb{R}^{k}\right)$-rectifiability, and we prove that Euclidean $k$-codimensional rectifiable sets are $\left(\mathbb{G}, \mathbb{R}^{k}\right)$-rectifiable.
\end{abstract}

Keywords. Stratified groups, characteristic points, perimeter measure, Hausdorff measure

\section{Introduction}

The project of developing analysis in metric spaces is receiving remarkable attention in the last few years, with several monographs on this topic [7], [18], [35], [36], [64]. The class of Carnot-Carathéodory spaces provides good models to be studied in this perspective. They are characterized as connected differentiable manifolds with a family of smooth vector fields $X_{1}, \ldots, X_{m}$ which satisfy a Lie bracket generating condition. Under this assumption the "Carnot-Carathéodory distance" can be defined [33].

We will focus our attention on stratified groups, also known as Carnot groups [25], [59], which form a special class of Carnot-Carathéodory spaces. An important feature of stratified groups is the existence of a family of intrinsic dilations which scale well with the Carnot-Carathéodory distance and define the Hausdorff dimension of the group.

Stratified groups and Carnot-Carathéodory spaces have been thoroughly investigated in several different areas of analysis including optimal control theory, differential geometry, harmonic analysis and PDEs. Some relevant books are [10], [25], [34], [53], [66]. The systematic study of geometric measure theory on stratified groups has begun only

V. Magnani: Dipartimento di Matematica, via Buonarroti 2, I-56127 Pisa, Italy; e-mail:magnani@dm.unipi.it

Mathematics Subject Classification (2000): Primary 22E25; Secondary 22E30, 28A75, 53C17 
very recently with a continuously growing literature and it is now the subject of several contributions: [1], [5], [8], [12], [15], [17], [26]-[30], [32], [41], [45]-[48], [54], [57], [60]-[62], [67], and this list is certainly not complete.

The leading theme of this paper is the role of characteristic points in the study of intrinsic notions of rectifiability and in the expression of perimeter measure for open sets with $C^{1}$ boundary. In the area of PDEs, characteristic points have been widely studied in connection with boundary regularity of solutions to equations with nonnegative characteristic form [11], [13], [21], [24], [38], [39], [42]-[44] (see also the references therein). The solution to the Dirichlet problem for the Kohn Laplacian in a smooth domain may not be smooth up to the boundary due to the presence of characteristic points [38]. Characteristic points play an important role in connection with metric properties of domains suitable for Sobolev-Poincaré inequality, Ahlfors regularity of hypersurfaces, intrinsic measure of hypersurfaces and trace theorems [8], [12], [15], [16], [22], [31], [48], [55], [56].

$\mathrm{W}$ now give a detailed description of the content of the present paper. The first section presents all the basic material that will be used throughout the paper. Section 2 is devoted to characteristic points of submanifolds and their negligibility. Negligibility of characteristic points with respect to the Euclidean Hausdorff measure $\mathcal{H}_{1 .}^{n-1}$ was proved by Derridj for $(n-1)$-dimensional smooth hypersurfaces [22]. Recently, Balogh through a remarkable construction of functions with prescribed gradient has proved that there exist $C^{1}$ hypersurfaces in the Heisenberg group such that the one-codimensional Euclidean Hausdorff measure of the characteristic set is positive [8]. However, he also shows that if we consider the Hausdorff measure $\mathcal{H}^{Q-1}$ with respect to the CarnotCarathéodory distance, then the characteristic set of $C^{1}$ hypersurfaces becomes negligible with respect to this measure. The covering type procedure adopted by Balogh has been extended by Franchi, Serapioni and Serra Cassano to all stratified groups of step two [29].

Our approach to the negligibility of characteristic points in arbitrary stratified groups relies on a Sard-type theorem [47], where characteristic points are properly interpreted as intrinsic singular points of the submanifold. This characterization works for submanifolds of any codimension and allows us to find an analogous notion of characteristic point. In this general case, we call these points "horizontal", because the tangent space to the submanifold at these points has a sufficiently "large" intersection with the horizontal space (see Definition 2.10p. Note that in submanifolds of codimension one the notion of horizontal point coincides with the classical notion of characteristic point.

The main result of this paper is given in Theorem 2.16, where we show that the set of horizontal points in a $k$-codimensional submanifold is $\mathcal{H}^{Q-k}$-negligible. The key observation is to look at horizontal points as those points where the defining map of the surface is "intrinsically singular", namely, it has a nonsurjective P-differential (Definition 2.2. Once horizontal points are regarded as singular points we construct an $a d$ hoc argument in order to apply the weak Sard-type theorem proved in [47], due to which a.e. level set of a vector-valued Lipschitz map contains an $\mathcal{H}^{Q-k}$-negligible subset of singular points. The negligibility of horizontal points in stratified groups immediately extends the validity of Theorem 2.3 in [48] to any $C^{1}$ hypersurface. Let $\Sigma$ be a $C^{1}$ hypersurface $\Sigma$ and let $\mathcal{S}^{Q-1}$ be the spherical Hausdorff measure with respect to a homogeneous distance. Then 
Theorem 2.20 gives an explicit formula for the density of $\mathcal{S}^{Q-1}\llcorner\Sigma$ with respect to the Riemannian measure restricted to the hypersurface.

In Section 3 we present other consequences of Theorem 2.16 We first introduce the notions of $\left(\mathbb{G}, \mathbb{R}^{k}\right)$-regularity and of $\left(\mathbb{G}, \mathbb{R}^{k}\right)$-rectifiability (Definitions 3.1 and 3.2. These notions for $k=1$ have been first introduced and studied by Franchi, Serapioni and Serra Cassano in the cycle of papers [27]-[29]. As the classical notion of differentiability is used to define the smoothness of a manifold, we observe that P-differentiability naturally defines $\left(\mathbb{G}, \mathbb{R}^{k}\right)$-regularity for every codimension, introducing a vast class of new geometrical objects to be studied, where $\left(\mathbb{G}, \mathbb{R}^{k}\right)$-regular sets play the role of "intrinsic regular submanifolds". However, those groups $\mathbb{G}$ whose Lie algebra $\mathcal{G}$ does not contain any $k$ dimensional commutative subalgebra in the first layer cannot have $\left(\mathbb{G}, \mathbb{R}^{k}\right)$-regular sets. Clearly, an $\left(\mathbb{R}^{q}, \mathbb{R}^{k}\right)$-regular set is exactly a $C^{1}$ submanifold of codimension $k$. On the other hand, as soon as we consider a noncommutative stratified group, things can dramatically change. A recent paper by Kirchheim and Serra Cassano [41] shows a remarkable example of an $\left(\mathbb{H}^{1}, \mathbb{R}\right)$-regular set $S$ such that $\mathcal{H}_{|\cdot|}^{\alpha}(S)>0$, where $|\cdot|$ is the Euclidean norm and $2<\alpha<5 / 2$, where $\mathbb{H}^{1}$ is the three-dimensional Heisenberg group. This interesting hypersurface cannot be 2-rectifiable in the Euclidean sense, although there exists a 1/2-Hölder continuous parametrization which makes it a topological submanifold of topological dimension two. Recently, $\left(\mathbb{H}^{n}, \mathbb{R}^{k}\right)$-regular sets, with $1 \leq k \leq n$, have been studied by Franchi, Serapioni and Serra Cassano [30]. In the terminology of [30], these sets are called $k$-codimensional $\mathbb{H}$-regular surfaces. Note that there are no $\left(\mathbb{H}^{n}, \mathbb{R}^{k}\right)$ regular sets when $k>n$.

Our negligibility result applies in comparing $\left(\mathbb{G}, \mathbb{R}^{k}\right)$-rectifiability with Euclidean rectifiability in the Federer sense (3.2.14 of [23]). In the papers [27] and [29] Franchi, Serapioni and Serra Cassano have proved that Euclidean rectifiable sets of codimension one in stratified groups of step two are always $(\mathbb{G}, \mathbb{R})$-rectifiable. As application of Theorem 2.16 in Theorem 3.8 we extend this result to any Euclidean rectifiable set of any stratified group. Note that horizontal curves or more general Legendrian submanifolds cannot be $\left(\mathbb{G}, \mathbb{R}^{k}\right)$-regular. In fact, from the notion of horizontal point, one can check that any $k$-codimensional Legendrian submanifold coincides with its horizontal set, so Theorem 2.16 proves that it is $\mathcal{H}^{Q-k}$-negligible. In other words, a $k$-codimensional Legendrian submanifold is invisible in a $\left(\mathbb{G}, \mathbb{R}^{k}\right)$-rectifiable set in the same way as Euclidean $(q-k-s)$-rectifiable sets are invisible in a Euclidean $(q-k)$-rectifiable set, where $s>0$. It is certainly interesting to investigate which couples $\left(\mathbb{G}, \mathbb{R}^{k}\right)$ possess nontrivial $\left(\mathbb{G}, \mathbb{R}^{k}\right)$-regular sets and to check whether these sets have Hausdorff dimension $Q-k$ and topological dimension $q-k$, where $q$ is the topological dimension of $\mathbb{G}$. Presently, this question has been positively answered for $k=1$ and arbitrary $\mathbb{G}$ in [28] and for $k \leq n$ and $\mathbb{G}=\mathbb{H}^{n}$ in [30]. Expanding our perspective, it is natural to replace $\mathbb{R}^{k}$ with another stratified group $\mathbb{M}$, getting the general notion of $(\mathbb{G}, \mathbb{M}$ )-regularity (Definition 3.5). We introduce this notion of regularity to illustrate its potential in connection with future developments of geometric measure theory on stratified groups. These notions of intrinsic regularity provide several types of rectifiable objects modelled with respect to different geometries. This shows how the geometry of stratified groups is rich and is still far from being well understood. 
Our negligibility result can also be applied to study the relationship between the perimeter measure of a set with $C^{1}$ boundary and the spherical Hausdorff measure of its boundary. In the more general case when a portion $\partial E \cap U$ of $\partial E$ is a $(\mathbb{G}, \mathbb{R})$-regular set and $U$ is an open subset, the formula

$$
|\partial E|_{H}\left\llcorner U=\theta_{Q-1}^{g}\left(v_{H}\right) \mathcal{S}^{Q-1}\llcorner(U \cap \partial E)\right.
$$

can be found in [28] and [49]. Due to $\mathcal{H}^{Q-1}$-negligibility of characteristic points this formula holds for any set $E$ with $C^{1}$ boundary and any measurable set of $\mathbb{G}$. As a consequence, we can positively answer a conjecture raised by Danielli, Garofalo and Nhieu in [15]. A detailed discussion of this application will be given in the last part of Section 3

\section{Preliminaries}

We will mean by measure on a metric space $X$ a set function $\mu: \mathcal{P}(X) \rightarrow[0,+\infty]$ with the properties $\mu(\emptyset)=0$ and $\mu(E) \leq \sum_{j=1}^{\infty} \mu\left(E_{j}\right)$ whenever $E \subset \bigcup_{j=1}^{\infty} E_{j}$. Every measure $\mu$ naturally induces a $\sigma$-algebra $\mathcal{A}_{\mu} \subset \mathcal{P}(X)$ where it is additive on countable disjoint unions. Recall that $\mathcal{B}(X)$ is the smallest $\sigma$-algebra containing open sets of $X$. Elements of $\mathcal{B}(X)$ are called Borel sets and a measure $\mu$ such that $\mathcal{B}(X) \subset \mathcal{A}_{\mu}$ is called a Borel measure. The push-forward of a measure $\mu$ under the map $F: X \rightarrow Y$ is defined by $F_{\sharp} \mu(E)=\mu\left(F^{-1}(E)\right)$ for every $E \subset Y$. The restriction of a measure $\mu$ to a subset $A \subset X$ is defined by $\mu\llcorner A(E)=\mu(A \cap E)$ for every $E \subset X$. The following elementary change of variable formula will be useful. Its proof can be obtained by approximation of measurable functions with measurable step functions.

Proposition 1.1 (Change of variable). Let $X, Y$ be two metric spaces and let $N$ be either $\overline{\mathbb{R}}$ or a finite-dimensional space. Suppose that $F: X \rightarrow Y$ and $u: Y \rightarrow N$ are Borel maps, where $\mu$ is a Borel measure over $X$ and $u \circ F$ is $\mu$-summable. Then for every $B \in \mathcal{B}(X)$ we have

$$
\int_{F^{-1}(B)} u \circ F d \mu=\int_{B} u d F_{\sharp} \mu .
$$

Definition 1.2 (Stratified group). Let $\mathbb{G}$ be a simply connected Lie group and let $\mathcal{G}$ be its Lie algebra of left invariant vector fields. Let $H^{1}$ be a subspace of $\mathcal{G}$ and suppose that the inductively defined sequence of subspaces

$$
H^{j+1}=\left[H^{j}, H^{1}\right]=\operatorname{span}\left\{[Z, Y] \mid Z \in H^{j}, Y \in H^{1}\right\}
$$

has the properties:

(1) $H^{j} \cap H^{k}=\{0\}$ whenever $j \neq k$ and $j, k \in \mathbb{N} \backslash\{0\}$,

(2) there exists $\iota \geq 1$ such that $H^{\iota} \neq\{0\}$ and $H^{\iota+1}=\{0\}$,

(3) $\mathcal{G}$ is spanned by all the subspaces $\left\{H^{j} \mid j=1, \ldots, \iota\right\}$. 
We say that $\mathcal{G}$ is a stratified algebra and that $\mathbb{G}$ is a stratified group. The integer is called the step of the group. A left translation of the group is a map $l_{p}: \mathbb{G} \rightarrow \mathbb{G}$ defined by $s \mapsto l_{p}(s)=p s$, where $p, s \in \mathbb{G}$. As a consequence of (1) and (3) every vector $Y \in \mathcal{G}$ can be written in a unique way as the sum $Y=\sum_{j=1}^{\iota} Y_{j}$, where $Y_{j} \in H^{j}$. This enables us to introduce canonical projections $\mathfrak{p}_{H^{j}}: \mathcal{G} \rightarrow H^{j}$ defined by $\mathfrak{p}_{H^{j}}(Y)=Y_{j}$, where $Y$ is written as above. For the projection on the first layer we also write $\mathfrak{p}_{H}=\mathfrak{p}_{H^{1}}$.

Conditions (1) and (3) of the previous definition can be briefly stated by writing

$$
\mathcal{G}=H^{1} \oplus \cdots \oplus H^{\iota}
$$

where the symbol $\oplus$ indicates the direct sum of vector spaces.

Recall that the exponential map exp $: \mathcal{G} \rightarrow \mathbb{G}$ associates to any left invariant vector field $W \in \mathcal{G}$ the value $\gamma_{W}(1) \in \mathbb{G}$ of the curve $\gamma_{W}: \mathbb{R} \rightarrow \mathbb{G}$ which solves the Cauchy problem

$$
\left\{\begin{array}{l}
\gamma^{\prime}(t)=W(\gamma(t)), \\
\gamma(0)=e .
\end{array}\right.
$$

We will use the fact that the exponential map exp : $\mathcal{G} \rightarrow \mathbb{G}$ of a simply connected nilpotent Lie group is a diffeomorphism [14].

Definition 1.3 (Graded metric). We say that a left invariant Riemannian metric $g$ on $\mathbb{G}$ is a graded metric if all subspaces $\left\{H^{j} \mid j=1, \ldots, \iota\right\}$ are orthogonal to each other. The left invariant scalar product between two vectors $V, W \in T_{p} \mathbb{G}$ will be denoted by $\langle V, W\rangle_{p}$ or $\langle V(p), W(p)\rangle$. In the case $X, Y \in \mathcal{G}$ the scalar product $\langle X, Y\rangle_{p}$ is independent of the point $p$ and is simply denoted by $\langle X, Y\rangle$.

Definition 1.4 (Graded coordinates). We define the numbers $n_{j}=\operatorname{dim} H^{j}$ for any $j=$ $1, \ldots, \iota, m_{0}=0$ and $m_{i}=\sum_{j=1}^{i} n_{j}$ for any $i=1, \ldots, \iota$. We say that a basis $\left(W_{1}, \ldots, W_{q}\right)$ of $\mathcal{G}$ is an adapted basis if $\left(W_{m_{j-1}+1}, W_{m_{j-1}+2}, \ldots, W_{m_{j}}\right)$ is a basis of $H^{j}$ for any $j=$ $1, \ldots, \iota$. We say that $\left(W_{1}, \ldots, W_{q}\right)$ is a graded basis if it is an adapted and orthonormal basis with respect to a graded metric. The graded coordinates with respect to the basis $\left(W_{1}, \ldots, W_{q}\right)$ are given by the diffeomorphism $F: \mathbb{R}^{q} \rightarrow \mathbb{G}$ defined by

$$
F(x)=\exp \left(\sum_{j=1}^{q} x_{j} W_{j}\right) .
$$

The degree of the coordinate $x_{j}$ is the unique integer $d_{j}$ such that $W_{j} \in H^{d_{j}}$.

Let $X, Y \in \mathcal{G}$. The Baker-Campbell-Hausdorff formula (briefly BCH-formula) allows us to obtain an explicit polynomial $P(X, Y)$ with respect to the nonassociative Lie product of $\mathcal{G}$ such that $\exp (P(X, Y))=\exp X \exp Y$ (see for instance [37]). The BCH-formula and graded coordinates allow us to view the group operation in $\mathbb{R}^{q}$ as a polynomial operation (which depends on the fixed system of graded coordinates). We will denote this polynomial operation by $x \cdot y$, where $x, y \in \mathbb{R}^{q}$. In case the Lie algebra $\mathcal{G}$ is commutative, i.e. $[X, Y]=0$ for every $X, Y \in \mathcal{G}$, we simply have $x \cdot y=x+y$ and all coordinates have degree one (see also Chapter XIII, Section 5 of [65]). 
Proposition 1.5. Let $F: \mathbb{R}^{q} \rightarrow \mathbb{G}$ be a system of graded coordinates with respect to a graded metric g. Then $F_{\sharp} \mathcal{L}^{q}=v_{g}$, where $\mathcal{L}^{q}$ is the q-dimensional Lebesgue measure.

Proof. We know that $F: \mathbb{R}^{q} \rightarrow \mathbb{G}$ is a smooth diffeomorphism. Let $A$ be a measurable subset of $\mathbb{R}^{q}$. By the classical area formula and the left invariance of both $v_{g}$ and $F_{\sharp} \mathcal{L}^{q}$ we have

$$
c \mathcal{L}^{q}(A)=v_{g}(F(A))=\int_{A} J_{q} F(\xi) d \xi
$$

for some constant $c>0$. We have denoted by $J_{q} F$ the $q$-dimensional Jacobian of $F$. Then $f_{A} J_{q} F=c$ for any measurable $A$. By continuity of $\xi \mapsto J_{q} F(\xi)$ we infer that $J_{q} F(\xi)=c$ for any $\xi \in \mathbb{R}^{q}$. We know that $F=\exp \circ L$, where $L(\xi)=\sum_{j=1}^{q} \xi_{j} W_{j}$ and $\left(W_{j}\right)$ is an orthonormal basis of $\mathcal{G}$. Since the map $d F(0)=d \exp (0) \circ L=L$ has Jacobian equal to one, we have $c=1$ and the assertion follows.

Motivated by the previous proposition we will also adopt the simpler notation $v_{g}(A)=$ $|A|$ for every measurable subset $A \subset \mathbb{G}$.

Definition 1.6 (Dilations). Let $\mathbb{G}$ be a stratified group. For every $r>0$ we define the dilation $\delta_{r}: \mathbb{G} \rightarrow \mathbb{G}$ by

$$
\delta_{r} p=\exp \left(\sum_{j=1}^{\iota} r^{j} \mathfrak{p}_{H^{j}}\left(\exp ^{-1}(p)\right)\right) .
$$

Consider a system of graded coordinates $F: \mathbb{R}^{q} \rightarrow \mathbb{G}$. The coordinate dilation $\Lambda_{r}$ : $\mathbb{R}^{q} \rightarrow \mathbb{R}^{q}$ associated to $F$ is defined by $\Lambda_{r}=F^{-1} \circ \delta_{r} \circ F$ and can be written as

$$
\Lambda_{r}(x)=\sum_{j=1}^{q} r^{d_{j}} x_{j} e_{j},
$$

where $\left(e_{j}\right)$ is the canonical basis of $\mathbb{R}^{q}$ and $d_{j}$ is the degree of the coordinate $x_{j}$.

In view of relation 2 it is easy to see that $\mathcal{L}^{q}\left(\Lambda_{r}(A)\right)=r^{Q} \mathcal{L}^{q}(A)$ for any measurable subset $A \subset \mathbb{R}^{q}$, where we have defined

$$
Q=\sum_{k=1}^{q} d_{k}=\sum_{j=1}^{\iota} j \operatorname{dim}\left(H^{j}\right) .
$$

Thus, by definition of coordinate dilation and by Proposition 1.5 , for any $r>0$ and any measurable subset $E \subset \mathbb{G}$ we have

$$
v_{g}\left(\delta_{r} E\right)=v_{g}\left(F\left(\Lambda_{r}\left(F^{-1}(E)\right)\right)\right)=\mathcal{L}^{q}\left(\Lambda_{r}\left(F^{-1}(E)\right)\right)=r^{Q} \mathcal{L}^{q}\left(F^{-1}(E)\right)=r^{Q} v_{g}(E) .
$$

Finally, the left invariance of $v_{g}$ yields the formula

$$
v_{g}\left(l_{p} \circ \delta_{r}(E)\right)=r^{Q} v_{g}(E)
$$

for any $p \in \mathbb{G}$ and any $r>0$. Observing that $B_{p, r}=l_{p}\left(\delta_{r} B_{1}\right)$ we have in particular

$$
v_{g}\left(B_{p, r}\right)=r^{Q} v_{g}\left(B_{1}\right)
$$


This implies that the number $Q$ defined in 3 is the Hausdorff dimension of the group and that the $Q$-dimensional Hausdorff measure $\mathcal{H}^{Q}$ built with respect to a homogeneous distance is finite on bounded sets and it is proportional to $v_{g}$.

The horizontal subbundle $H \mathbb{G}$ is defined by the collection of all subspaces

$$
H_{p} \mathbb{G}=\left\{X(p) \mid X \in H^{1}\right\}
$$

where $p \in \mathbb{G}$. These are the so-called horizontal spaces. We denote by $H \Omega$ the subfamily of horizontal spaces $H_{p} \mathbb{G}$ where $p \in \Omega$ and $\Omega$ is an open subset of $\mathbb{G}$. Another way to introduce horizontal spaces is the following:

$$
d l_{p}\left(H_{e} \mathbb{G}\right)=H_{p} \mathbb{G}
$$

for every $p \in \mathbb{G}$, where $e$ is the unit element of the group.

By definition of stratified group, the Lie algebra spanned by $H^{1}$ coincides with $\mathcal{G}$, so the well known Chow theorem implies that any two points of $\mathbb{G}$ can be connected by at least one absolutely continuous curve a.e. tangent to the horizontal subbundle [10]. These curves are the so-called horizontal curves which permit us to introduce the CarnotCarathéodory distance. Let $p, p^{\prime} \in \mathbb{G}$ and consider the infimum of the lengths of all horizontal curves connecting $p$ with $p^{\prime}$, where the length is computed with respect to the graded metric of the group. This infimum is the Carnot-Carathéodory distance between $p$ and $p^{\prime}$ and it is denoted by $\rho\left(p, p^{\prime}\right)$. The left invariance of the graded metric implies that the Carnot-Carathéodory distance is left invariant, that is, $\rho\left(p^{\prime} p, p^{\prime} s\right)=\rho(p, s)$ for every $p, p^{\prime}, s \in \mathbb{G}$, and it is also homogeneous with respect to dilations, $\rho\left(\delta_{r} p, \delta_{r} s\right)=$ $r \rho(p, s)$ for every $r>0$.

Next we will use the usual Euclidean norm on $\mathbb{G}$. To do this, there is not a unique choice and we will refer to a fixed system of graded coordinates $F: \mathbb{R}^{q} \rightarrow \mathbb{G}$. This choice will not affect our arguments because if $G: \mathbb{R}^{q} \rightarrow \mathbb{G}$ is another system of graded coordinates, the change of variable $F \circ G^{-1}: \mathbb{R}^{q} \rightarrow \mathbb{R}^{q}$ is an isomorphism, hence the Euclidean norm with respect to $G$ is equivalent to the one with respect to $F$. With this convention we state an important relation between the Euclidean distance and the CarnotCarathéodory distance on stratified groups:

$$
|x-y| \leq \rho(x, y) \leq C|x-y|^{1 / \iota} \quad \text { for any } x, y \in K \subset \mathbb{G},
$$

where $K$ is compact and $C$ is a dimensional constant depending on $K$. Note that $\iota$ is the step of the group. Estimates (6) can be proved in more general Carnot-Carathéodory spaces [58]. In particular the Carnot-Carathéodory distance is continuous and it induces the same topology of $\mathbb{G}$.

Definition 1.7 (Homogeneous distance). A continuous map $d: \mathbb{G} \times \mathbb{G} \rightarrow[0,+\infty)$ is $a$ homogeneous distance if it satisfies the axioms of an abstract distance, it is left invariant, $d\left(p^{\prime} p, p^{\prime} s\right)=d(p, s)$ for any $p, p^{\prime}, s \in \mathbb{G}$, and it is homogeneous with respect to dilations, $d\left(\delta_{r} p, \delta_{r} s\right)=r d(p, s)$ for every $r>0$. 
Clearly the Carnot-Carathéodory distance is the foremost homogeneous distance, but also other useful examples of homogeneous distances can be considered. For instance, in Proposition 2.19 we will study the symmetry properties of the homogeneous distance $d_{\infty}$ introduced in [29]. It is worth observing that any two homogeneous distances are biLipschitz equivalent in the following sense. If $d_{1}$ and $d_{2}$ are homogeneous distances, then there exists a constant $C>1$ such that for every $p, s \in \mathbb{G}$ we have

$$
C^{-1} d_{1}(p, s) \leq d_{2}(p, s) \leq C d_{1}(p, s)
$$

This fact can be seen using the classical argument used for norms of finite-dimensional spaces, which actually is a particular case. In what follows we will always refer to a fixed homogeneous distance.

Definition 1.8 (Metric ball). The open ball of radius $r>0$ and centre $p \in \mathbb{G}$ will be denoted by $B_{p, r}$. If we wish to emphasize the particular choice of the homogeneous distance $d$ we write $B_{p, r}^{d}$. Open balls with radius $r$ and centred at the unit element of the group are denoted by $B_{r}$. We use similar conventions for closed balls $D_{p, r}$ of centre $p$ and radius $r$.

Definition 1.9 (Hausdorff measures). Let $d$ be a homogeneous distance of $\mathbb{G}$ and let $a \geq 0$. For each subset $E \subset \mathbb{G}$ we define the a-dimensional spherical Hausdorff measure

$$
\mathcal{S}^{a}(E)=\lim _{\varepsilon \rightarrow 0^{+}} \inf \left\{\sum_{i=1}^{\infty} \frac{\operatorname{diam}\left(D_{x_{i}, t_{i}}\right)^{a}}{2^{a}} \mid E \subset \bigcup_{i=1}^{\infty} D_{x_{i}, t_{i}}, t_{i} \leq \varepsilon\right\}
$$

and the a-dimensional Hausdorff measure as

$$
\mathcal{H}^{a}(E)=\lim _{\varepsilon \rightarrow 0^{+}} \inf \left\{\sum_{i=1}^{\infty} \omega_{a} \frac{\operatorname{diam}\left(F_{i}\right)^{a}}{2^{a}} \mid E \subset \bigcup_{i=1}^{\infty} F_{i}, \operatorname{diam}\left(F_{i}\right) \leq \varepsilon\right\}
$$

where $\left\{F_{i}\right\}$ are subsets of $\mathbb{G}$ and $\operatorname{diam}(A)=\sup _{(x, y) \in A \times A} d(x, y)$ for any $A \subset \mathbb{G}$. The dimensional constant $\omega_{a}$ is defined as follows:

$$
\omega_{a}=\frac{\pi^{a / 2}}{\Gamma(1+a / 2)}, \quad \Gamma(s)=\int_{0}^{\infty} r^{s-1} e^{-r} d r .
$$

Remark 1.10. Note that we have used the dimensional constant $\omega_{a}$ only for the definition of the Hausdorff measure $\mathcal{H}^{a}$. In fact, our formulae involving the spherical Hausdorff measure $\mathcal{S}^{a}$ will contain the metric factor $\theta_{Q-1}^{g}$ (Definition 2.17) that naturally replaces the constant $\omega_{a}$.

When we want to specify the use of the Euclidean distance we will write $\mathcal{H}_{|\cdot|}^{a}$ and $\mathcal{S}_{|.|}^{a}$, and for the case of the Carnot-Carathéodory distance we will write $\mathcal{H}_{\rho}^{a}$ and $\mathcal{S}_{\rho}^{a}$. Throughout the paper the symbol $\Omega$ will denote an open subset of $\mathbb{G}$. We will denote by $\Gamma(H \Omega)$ the space of smooth sections of $H \Omega$, i.e. the horizontal vector fields. The symbol $\Gamma_{c}(H \Omega)$ denotes the family of horizontal vector fields compactly supported in $\Omega$. 
Definition 1.11 (Horizontal divergence). Let $\left(X_{1}, \ldots, X_{m}\right)$ be a basis of left invariant vector fields of $H^{1}$. The horizontal divergence (for short, $H$-divergence) of the section $\varphi=\sum_{i=1}^{m} \varphi^{j} X_{j} \in \Gamma(H \Omega)$ is defined by $\operatorname{div}_{H} \varphi=\sum_{j=1}^{m} X_{j} \varphi^{j}$.

Notice that this definition does not depend on the choice of the basis of $H^{1}$. Now we recall the notion of "image" of a vector field through a diffeomorphism. Let $f: M \rightarrow N$ be a $C^{1}$ diffeomorphism of differentiable manifolds and let $X$ be a vector field on $M$. The image of $X$ under $f$ is the vector field on $N$ defined by

$$
f_{*} X(p)=d f\left(f^{-1}(p)\right)\left(X\left(f^{-1}(p)\right)\right)
$$

for every $p \in N$. A vector field $X \in H^{1}$ is viewed in $\mathbb{R}^{q}$ through a system of graded coordinates $F: \mathbb{R}^{q} \rightarrow \mathbb{G}$ when it is defined as $\tilde{X}=F_{*}^{-1} X$. We will use this notation to denote vector fields of $\mathcal{G}$ with respect to graded coordinates of $\mathbb{R}^{q}$. With this notation the horizontal divergence can be written as follows:

$$
\left(\operatorname{div}_{H} \varphi\right) \circ F=\sum_{j=1}^{m} \tilde{X}_{j} \tilde{\varphi}^{j},
$$

where $\varphi \in \Gamma(H \mathbb{G})$ and $\tilde{\varphi}=\varphi \circ F$. Another useful formula involving left invariant vector fields in $\mathbb{R}^{q}$ is

$$
\tilde{W}_{k}=\partial_{x_{k}}+\sum_{j=m_{d_{k}}+1}^{q} a_{k j}\left(x_{1}, \ldots, x_{j-1}\right) \partial_{x_{j}},
$$

where $\tilde{W}_{k}=F_{*}^{-1} W_{k}$ with $k=1, \ldots, q$, the integers $m_{i}$ and $d_{j}$ are introduced in Definition 1.4 the maps $a_{k j}$ are homogeneous polynomials with respect to coordinate dilations and the graded basis $\left(W_{1}, \ldots, W_{q}\right)$ of $\mathcal{G}$ is associated to the system of graded coordinates $F$ (see also p. 621 of [65]). Note that in formula (8] we have used the standard representation of vector fields as first order differential operators.

Let $u: \Omega \rightarrow \mathbb{R}$ be a $C^{1}$ map, $p=F(x) \in \Omega$ and $\tilde{u}=u \circ F$. We consider the map $y \mapsto \tilde{u}(x \cdot y)$. By left invariance of the vector fields $\tilde{W}_{j}$ on $\mathbb{R}^{q}$ we have the useful formula

$$
\left.\frac{\partial \tilde{u}}{\partial y_{j}}\right|_{y=0}=\tilde{W}_{j} \tilde{u}(x)=W_{j} u(p) .
$$

In the following definition the symbol $|\cdot|$ will denote the norm induced by the Riemannian metric on tangent spaces. We will use this notation whenever its meaning is clear from the context.

Definition 1.12 (Perimeter measure). We say that a measurable set $E \subset \Omega$ is of $\mathrm{H}$ finite perimeter on the open subset $\Omega \subset \mathbb{G}$ if

$$
|\partial E|_{H}(\Omega)=\sup \left\{\int_{E} \operatorname{div}_{H} \phi d v_{g}\left|\phi \in \Gamma_{c}(H \Omega),\right| \phi \mid \leq 1\right\}<\infty .
$$

If $E$ has $H$-finite perimeter on every open subset $U$ compactly contained in $\Omega$ we say that $E$ has locally $H$-finite perimeter in $\Omega$. We will denote by $|\partial E|_{H}$ the associated Radon measure. 
By the Riesz representation theorem there exists a measurable section $v_{E}$ of $H \mathbb{G}$ such that

$$
\int_{E} \operatorname{div}_{H} \phi d v_{g}=-\int_{\mathbb{G}}\left\langle\phi, v_{E}\right\rangle d|\partial E|_{H}
$$

for any $\phi \in \Gamma_{c}(H \mathbb{G})$. We say that $v_{E}$ is the generalized inward normal to $E$. By the standard polar decomposition (Corollary 1.29 of [3]) we have $\left|v_{E}(p)\right|=1$ for $|\partial E|_{H}$-a.e. $p \in \Omega$.

\section{Horizontal set of $C^{1}$ submanifolds}

In this section we introduce the notion of horizontal point for a $k$-codimensional submanifold and we prove that the set of all horizontal points is $\mathcal{H}^{Q-k}$-negligible. The symbols $\mathbb{G}$ and $\mathbb{M}$ denote stratified groups and $\Omega$ stands for an open subset of $\mathbb{G}$. We will show the relationship between the intrinsic notion of P-differentiability and the notion of horizontal point. Recall that P-differentiability of maps between stratified groups was successfully introduced by Pansu in order to study rigidity properties [59].

Definition 2.1 (G-linear map). We say that $L: \mathbb{G} \rightarrow \mathbb{M}$ is a G-linear map if it is a group homomorphism and $L\left(\delta_{r} p\right)=\delta_{r}^{\prime} L(p)$ for every $p \in \mathbb{G}$ and every $r>0$, where $\delta_{r}$ and $\delta_{r}^{\prime}$ are dilations of the stratified groups $\mathbb{G}$ and $\mathbb{M}$, respectively.

Definition 2.2 (P-differentiable map). Let $f: \Omega \rightarrow \mathbb{M}$, where $\mathbb{M}$ is a stratified group. We say that $f$ is $\mathrm{P}$-differentiable at $p \in \Omega$ if there exists a G-linear map $L: \mathbb{G} \rightarrow \mathbb{M}$ such that

$$
\frac{\rho^{\prime}\left(f(p)^{-1} f(s), L\left(p^{-1} s\right)\right)}{\rho(p, s)} \rightarrow 0 \quad \text { as } s \rightarrow p,
$$

where $\rho$ and $\rho^{\prime}$ are the Carnot-Carathéodory distances of $\mathbb{G}$ and $\mathbb{M}$, respectively. The unique G-linear map which satisfies (11) is called the P-differential of $f$ at $p$ and it is denoted by $d_{H} f(p)$. Via the exponential map exp $: \mathcal{G} \rightarrow \mathbb{G}$ we will also view the $P$ differential as a linear map between the Lie algebras of $\mathbb{G}$ and of $\mathbb{M}$. In this case we will use the same notation $d_{H} f(p)$.

Definition $2.3\left(C_{H}^{1}\right.$ map). We denote by $C_{H}^{1}(\Omega, \mathbb{M})$ the class of maps $f: \Omega \rightarrow \mathbb{M}$ which are $P$-differentiable at every point of $\Omega$ and whose P-differential $p \mapsto d_{H} f(p)$ is continuous. In the case $\mathbb{M}=\mathbb{R}$ we simply write $C_{H}^{1}(\Omega)$.

In the present paper we work with $C^{1}$ maps and $C^{1}$ submanifolds, but mainly using the notion of P-differentiability. The next proposition ensures that this is possible since $C^{1}$ maps in the usual sense are also $C_{H}^{1}$.

Proposition 2.4 (P-differentiability). The inclusion $C^{1}\left(\Omega, \mathbb{R}^{k}\right) \subset C_{H}^{1}\left(\Omega, \mathbb{R}^{k}\right)$ holds and for every $u \in C^{1}\left(\Omega, \mathbb{R}^{k}\right)$ and every $p \in \Omega$ we have the formula $d_{H} u(p)(Y(p))=$ $d u(p)\left(\mathfrak{p}_{H}(Y)(p)\right)$ whenever $Y \in \mathcal{G}$. The map $\mathfrak{p}_{H}: \mathcal{G} \rightarrow H^{1}$ is the canonical projection introduced in Definition 1.2 
Proof. Let us fix a system of graded coordinates $F: \mathbb{R}^{q} \rightarrow \mathbb{G}$ with respect to a graded basis $\left(W_{1}, \ldots, W_{q}\right)$ of $\mathcal{G}$. Define $\tilde{u}=u \circ F \in C^{1}\left(\tilde{\Omega}, \mathbb{R}^{k}\right)$, where $\tilde{\Omega}=F^{-1}(\Omega)$. We define $x=F^{-1}(p)$ where $p \in \Omega$. Using graded coordinates together with coordinate dilations and the notion of P-differentiability our proof boils down to showing the existence of the limit

$$
\lim _{r \rightarrow 0^{+}} \frac{\tilde{u}\left(x \cdot \Lambda_{r}(y)\right)-\tilde{u}(x)}{r}
$$

uniformly as $y$ varies in some bounded neighbourhood of the origin. Let us define the map

$$
r \mapsto \tilde{u}\left(x \cdot \Lambda_{r}(y)\right)=\tilde{u}\left(x \cdot\left(\sum_{j=1}^{q} r^{d_{j}} y_{j} e_{j}\right)\right)=\psi(r, y) .
$$

Since the map $\psi$ is $C^{1}$ we can write

$$
\frac{\psi(r, y)-\psi(0, y)}{r}=r^{-1} \int_{0}^{r} \partial_{t} \psi(\tau, y) d \tau,
$$

hence formula (9) implies that

$$
\begin{aligned}
& \frac{\psi(r, y)-\psi(0, y)}{r}=r^{-1} \sum_{j=1}^{q} \int_{0}^{r} d_{j} \tau^{d_{j}-1} y_{j} \tilde{W}_{j} \tilde{u}\left(x \cdot \Lambda_{\tau} y\right) d \tau \\
& =\sum_{j=1}^{m} y_{j} \int_{0}^{1} \tilde{W}_{j} \tilde{u}\left(x \cdot \Lambda_{r \tau} y\right) d \tau+\sum_{j=m+1}^{q} d_{j} r^{d_{j}-1} y_{j} \int_{0}^{1} \tau^{d_{j}-1} \tilde{W}_{j} \tilde{u}\left(x \cdot \Lambda_{r \tau} y\right) d \tau,
\end{aligned}
$$

where the degree $d_{j}$ of the coordinate $y_{j}$ is greater than one if and only if $j>m$. By the continuity of $\tilde{W}_{j} \tilde{u}_{j}$ for every $j=1, \ldots, q$, formula 9 and the last equality we have proved that

$$
\lim _{r \rightarrow 0^{+}} \frac{\tilde{u}\left(x \cdot \Lambda_{r}(y)\right)-\tilde{u}(x)}{r}=\sum_{j=1}^{m} y_{j} \tilde{W}_{j} \tilde{u}(x)=\sum_{j=1}^{m} y_{j} W_{j} u(p) .
$$

This expression yields a G-linear map with respect to graded coordinates $y$,

$$
d_{H} u(p)\left(\sum_{j=1}^{q} y_{j} W_{j}(p)\right)=\sum_{j=1}^{m} y_{j} W_{j} u(p) .
$$

The explicit formula for the P-differential and the continuity of $W_{j}$ for every $j=1, \ldots, q$ yield the continuity of $d_{H} u(p)$ with respect to $p$. Finally, observing that

$$
\mathfrak{p}_{H}\left(\sum_{j=1}^{q} y_{j} W_{j}\right)(p)=\sum_{j=1}^{m} y_{j} W_{j}(p) \in H_{p} \mathbb{G}
$$

for every $Y \in \mathcal{G}$ we obtain the relation $d_{H} u(p)(Y(p))=d u(p)\left(\mathfrak{p}_{H}(Y)(p)\right)$. 
Proposition 2.5. Let $u \in C^{1}(\Omega)$ and $p \in \Omega$. Then there exists a unique horizontal vector, denoted by $\nabla_{H} u(p) \in H_{p} \mathbb{G}$, such that $d_{H} u(p)(V)=\left\langle\nabla_{H} u(p), V\right\rangle_{p}$ for any $V \in T_{p} \mathbb{G}$ and $\nabla_{H} u(p)$ is the orthogonal projection of $\nabla u(p)$ onto $H_{p} \mathbb{G}$.

Proof. The linear map $d_{H} u(p)$ can be represented by a vector $W \in T_{p} \mathbb{G}$ such that $d_{H} u(p)(V)=\langle W, V\rangle_{p}$ for any $V \in T_{p} G$. We first prove that $W \in H_{p} \mathbb{G}$. Observe that $T_{p} \mathbb{G}=H_{p}^{1} \mathbb{G} \oplus \cdots \oplus H_{p}^{l} \mathbb{G}$ where we have defined, for any $j=1, \ldots, \iota$,

$$
H_{p}^{j} \mathbb{G}=\left\{X(p) \in T_{p} \mathbb{G} \mid X \in H^{j}\right\} .
$$

Then we can write $V=\sum_{j=1}^{\iota} V_{j}$ and $W=\sum_{j=1}^{\iota} W_{j}$, where $V_{j}, W_{j} \in H_{p}^{j} \mathbb{G}$. By Proposition 2.4 we know that $d_{H} u(p)(V)=d u(p)\left(V_{1}\right)=\left\langle\nabla u(p), V_{1}\right\rangle$, hence

$$
\left\langle\nabla u(p), V_{1}\right\rangle_{p}=\langle W, V\rangle_{p}=\left\langle W, V_{1}\right\rangle .
$$

Since we are using a graded metric the subspaces $\left\{H_{p}^{j} \mathbb{G} \mid j=1, \ldots, \iota\right\}$ are orthogonal to each other, so the arbitrary choice of $V$ implies that $W_{j}=0$ for every $2 \leq j \leq \iota$ and

$$
\left\langle\nabla u(p)-W_{1}, V_{1}\right\rangle=0
$$

for every $V_{1} \in H_{p} \mathbb{G}$. The last property characterizes $W_{1}$ as the orthogonal projection of $\nabla u(p)$ onto $H_{p} \mathbb{G}$. In particular $W_{1}$ is the projection of $W$ onto $H_{p} \mathbb{G}$. Thus, defining $\nabla_{H} u(p)=W_{1}$ completes the proof.

Definition 2.6 (Horizontal normal). Let $\Sigma \subset \Omega$ be a $C^{1}$ submanifold of codimension one and let $p \in \Sigma$. We denote by $\nu(p)$ a unit normal to $\Sigma$ at $p$ with respect to a fixed graded metric. We say that the orthogonal projection of $\nu(p)$ onto $H_{p} \mathbb{G}$ is a horizontal normal of $\Sigma$ at $p$ and we denote it by $v_{H}(p)$.

Remark 2.7. Note that the horizontal normal should be considered up to sign, because we do not require oriented submanifolds in the definition of horizontal normal, and the functions depending on the horizontal normal do not depend on its sign. In more rigorous terms, one should identify $v_{H}$ and $-v_{H}$ in $H_{p} \mathbb{G}$ and consider the corresponding quotient space.

Lemma 2.8. Let $u: \Omega \rightarrow \mathbb{R}$ be a $C^{1}$ map. Suppose that $u^{-1}(0) \neq \emptyset$ and assume that $\nabla u(p) \neq 0$ for every $p \in \Omega$. Then for every $p \in u^{-1}(0)$ and every $Z \in T_{p} \mathbb{G}$ we have

$$
d_{H} u(p)(Z)=|\nabla u(p)|\left\langle v_{H}(p), Z\right\rangle_{p} \quad \text { and } \quad v_{H}(p)=\frac{\nabla_{H} u(p)}{|\nabla u(p)|},
$$

where $v_{H}(p)$ is the horizontal normal of the submanifold $u^{-1}(0)$ at the point $p$.

Proof. We observe that $v(p)=\nabla u(p) /|\nabla u(p)|$ is a unit normal to $u^{-1}(0)$ at $p$. By definition of horizontal normal, the orthogonal projection of $v(p)$ onto $H_{p} \mathbb{G}$ is $v_{H}(p)$, and Proposition 2.5 concludes the proof. 
Definition 2.9 (Horizontal gradient). Let $u \in C^{1}(\Omega)$. In view of Proposition 2.5 we can define the horizontal gradient of $u$ as the unique $C^{1}$ horizontal vector field $\nabla_{H} u$ such that

$$
d_{H} u(p)(Y)=\left\langle\nabla_{H} u(p), Y\right\rangle_{p}
$$

for every $p \in \Omega$ and every $Y \in T_{p} \mathbb{G}$.

Definition 2.10 (Horizontal point). Let $\Sigma \subset \Omega$ be a $C^{1}$ submanifold of codimension $k$ and let $p \in \Sigma$. We say that $p \in \Sigma$ is a horizontal point of $\Sigma$ if

$$
\operatorname{dim}\left(H_{p} \mathbb{G}\right)-\operatorname{dim}\left(H_{p} \mathbb{G} \cap T_{p} \Sigma\right) \leq k-1 .
$$

The horizontal set of $\Sigma$ is the subset of all horizontal points and it is denoted by $C(\Sigma)$.

The last definition is inspired by Lemma 2.11 and Theorem 2.13 below, in the following sense. Let $\Sigma$ be a submanifold of codimension one defined as a level set of a $C^{1}$ map $u: \Omega \rightarrow \mathbb{R}$. Theorem 2.13 singles out a class of "intrinsic" singular points of $\Sigma$, corresponding to the subset of $\Sigma$ where $d_{H} u$ vanishes (this subset is well defined due to Proposition 2.4p. In view of Lemma 2.11 this subset exactly corresponds to the subset of horizontal points of $\Sigma$. The singularity of $u$ at $p \in \Sigma$ can also be expressed by the condition $\operatorname{dim}\left(H_{p} \mathbb{G}\right)=\operatorname{dim}\left(H_{p} \mathbb{G} \cap T_{p} \Sigma\right)$, that is, $d_{H} u(p)$ vanishes. For submanifolds of higher codimension condition 14 amounts to the nonsurjectivity of $d_{H} u(p): \mathbb{G} \rightarrow \mathbb{R}^{k}$, because $\operatorname{dim}\left(d_{H} u(p)\left(H_{p} \mathbb{G}\right)\right)=\operatorname{dim}\left(H_{p} \mathbb{G}\right)-\operatorname{dim}\left(H_{p} \mathbb{G} \cap T_{p} \Sigma\right)$, although the map $u$ does not appear in (14). In the case $k=1$, one can interpret inequality (14) via the inclusion $H_{p} \mathbb{G} \subset T_{p} \Sigma$, which coincides with the condition $\operatorname{dim}\left(H_{p} \mathbb{G}\right)=\operatorname{dim}\left(H_{p} \mathbb{G} \cap T_{p} \Sigma\right)$. It is clear that when $k>1$ condition (14) means that the horizontal subspace $H_{p} \mathbb{G}$ is allowed to intersect $T_{p} \Sigma$ without necessarily being contained in it. Thus in higher codimension, a point $p$ such that $H_{p} \mathbb{G} \subset T_{p} \Sigma$ is a horizontal point, but the horizontal set $C(\Sigma)$ includes a larger class of points.

Lemma 2.11 (Singular points). Let $O$ be an open subset of $\mathbb{G}$ containing the unit element $e$ and let $u \in C^{1}\left(O, \mathbb{R}^{k}\right)$ be such that $u(e)=0$ and the differential map $d u(p): T_{p} \mathbb{G} \rightarrow \mathbb{R}^{k}$ is surjective for any $p \in O$. Then, defining $\Sigma=u^{-1}(0)$, we represent the horizontal set of $\Sigma$ as follows:

$$
C(\Sigma)=\left\{p \in \Sigma \mid d_{H} u(p): \mathcal{G} \rightarrow \mathbb{R}^{k} \text { is not surjective }\right\} .
$$

Proof. We choose $p \in \Sigma \subset O$. Since $\Sigma$ is the level set $u^{-1}(0)$ we have $\operatorname{Ker}(d u(p))=$ $T_{p} \Sigma$. Define the subspace

$$
S(p)=\left\{X \in H^{1} \mid X(p) \in H_{p} \mathbb{G} \cap T_{p} \Sigma\right\} \subset H^{1} .
$$

We wish to prove that

$$
\operatorname{Ker}\left(d_{H} u(p)\right)=S(p) \oplus H^{2} \oplus \cdots \oplus H^{\iota} .
$$

We will use the formula

$$
d_{H} u(p)(Y(p))=d u(p)\left(\mathfrak{p}_{H}(Y)(p)\right) \quad \text { for any } Y \in \mathcal{G},
$$


proved in Proposition 2.4. Let $Y \in S(p) \oplus H^{2} \oplus \cdots \oplus H^{\iota}$. By definition of $S(p)$ we have $\mathfrak{p}_{H}(Y)(p) \in H_{p} \mathbb{G} \cap T_{p} \Sigma$ and by $\sqrt{16}$ we obtain $d_{H} u(p)\left(H^{j}\right)=\{0\}$ for every $j=2, \ldots, l$; thus $d_{H} u(p)(Y(p))=0$. As a result, we have proved the inclusion

$$
S(p) \oplus H^{2} \oplus \cdots \oplus H^{\iota} \subset \operatorname{Ker}\left(d_{H} u(p)\right) .
$$

Conversely, assume $Y \in \operatorname{Ker}\left(d_{H} u(p)\right)$. By $(16)$ it follows that $\mathfrak{p}_{H}(Y)(p) \in \operatorname{Ker}(d u(p))$, so $\mathfrak{p}_{H}(Y)(p) \in H_{p} \mathbb{G} \cap T_{p} \Sigma$. We have proved that $\mathfrak{p}_{H}(Y) \in S(P)$, hence $Y \in S(p) \oplus$ $H^{2} \oplus \cdots \oplus H^{\iota}$. This implies

$$
\operatorname{Ker}\left(d_{H} u(p)\right) \subset S(p) \oplus H^{2} \oplus \cdots \oplus H^{\iota},
$$

and (15) follows. The equalities $(15)$ and $\operatorname{dim}(S(p))=\operatorname{dim}\left(H_{p} \mathbb{G} \cap T_{p} \Sigma\right)$ yield

$$
\begin{aligned}
\operatorname{dim}\left(d_{H} u(p)(\mathcal{G})\right) & =\operatorname{dim}(\mathcal{G})-\operatorname{dim}\left(\operatorname{Ker}\left(d_{H} u(p)\right)\right) \\
& =\operatorname{dim}\left(H_{p} \mathbb{G}\right)-\operatorname{dim}\left(H_{p} \mathbb{G} \cap T_{p} \Sigma\right) .
\end{aligned}
$$

Now assume that $p \in C(\Sigma)$. By definition of horizontal point we know that

$$
\operatorname{dim}\left(H_{p} \mathbb{G}\right)-\operatorname{dim}\left(H_{p} \mathbb{G} \cap T_{p} \Sigma\right) \leq k-1,
$$

therefore formula (17) implies that $\operatorname{dim}\left(d_{H} u(p)(\mathcal{G})\right) \leq k-1$ and $d_{H} u(p): \mathcal{G} \rightarrow \mathbb{R}^{k}$ cannot be surjective. Conversely, if $d_{H} u(p): \mathcal{G} \rightarrow \mathbb{R}^{k}$ is not surjective, then by 17 it follows that $\operatorname{dim}\left(H_{p} \mathbb{G}\right)-\operatorname{dim}\left(H_{p} \mathbb{G} \cap T_{p} \Sigma\right) \leq k-1$. This concludes the proof.

Proposition 2.12. Let $\Sigma$ be a submanifold of $\mathbb{G}$. Then for any $p \in \mathbb{G}$ we have

$$
l_{p}(C(\Sigma))=C\left(l_{p}(\Sigma)\right) .
$$

Proof. The left translation $l_{p}: \mathbb{G} \rightarrow \mathbb{G}$ is a diffeomorphism, therefore $l_{p}(\Sigma)$ is another submanifold of $\mathbb{G}$. In view of $(5)$ and using the chain rule it follows that $d l_{p}(s)\left(H_{s} \mathbb{G}\right)=$ $H_{p s} \mathbb{G}$ for any $s \in \mathbb{G}$, hence

$$
d l_{p}(s)\left(H_{s} \mathbb{G} \cap T_{s} \Sigma\right)=H_{p s} \mathbb{G} \cap d l_{p}(s)\left(T_{s} \Sigma\right)=H_{p s} \mathbb{G} \cap T_{p s}\left(l_{p} \Sigma\right) .
$$

As a consequence, for any $s \in \mathbb{G}$ we have

$$
\operatorname{dim}\left(H_{s} \mathbb{G} \cap T_{s} \Sigma\right)=\operatorname{dim}\left(H_{p s} \mathbb{G} \cap T_{p s}\left(l_{p} \Sigma\right)\right) .
$$

By the definition of horizontal point, the last equality implies that $s \in C(\Sigma)$ if and only if $p s \in C\left(l_{p} \Sigma\right)$.

An essential tool to prove Theorem 2.16 below is the following Sard-type theorem, which corresponds to Theorem 2.7 of [47].

Theorem 2.13 (Sard-type theorem). Let $\mathbb{G}$ and $\mathbb{M}$ be stratified groups of Hausdorff dimension $Q$ and $P$, respectively, with $Q \geq P$. Let $A \subset \mathbb{G}$ be a measurable set. Consider a Lipschitz map $\tau: A \rightarrow \mathbb{M}$ and define the set of singular points

$$
S=\left\{p \in A \mid d_{H} \tau(p) \text { exists and it is not surjective }\right\} .
$$

Then $\mathcal{H}^{Q-P}\left(S \cap \tau^{-1}(\xi)\right)=0$ for $\mathcal{H}^{P}$-a.e. $\xi \in \mathbb{M}$. 
Remark 2.14. Due to the general Eilenberg inequality proved in 2.10 .25 of [23] the fact that the points where $u$ is not P-differentiable are negligible implies that they are $\mathcal{H}^{Q-P}$ negligible in $\mathcal{H}^{P}$-a.e. level set. In other words, for $\mathcal{H}^{P}$-a.e. level set of $u$ the subset of points $p$ where $d_{H} \tau(p)$ exists has full measure.

We will apply Theorem 2.13 to the case $\mathbb{M}=\mathbb{R}^{k}, P=k$ and $\tau \in C^{1}\left(U, \mathbb{R}^{k}\right)$, where $U$ is an open subset of $\mathbb{G}$. Due to Proposition 2.4 any map of $C^{1}\left(U, \mathbb{R}^{k}\right)$ also belongs to $C_{H}^{1}\left(U, \mathbb{R}^{k}\right)$, so it is everywhere P-differentiable, with continuous P-differential. The everywhere existence of the P-differential allows us to divide the points of $\tau^{-1}(t)$ into two disjoint subsets for every $t \in \mathbb{R}^{k}$. The first one is the subset of points $p \in \tau^{-1}(t)$ such that $d_{H} \tau(p): \mathcal{G} \rightarrow \mathbb{R}^{k}$ is not surjective, and the second one is the complement. It is clear that $C_{H}^{1}$ maps are locally Lipschitz with respect to the Carnot-Carathéodory distance, therefore Theorem 2.13 can be applied to $u_{\mid K}$, where $K$ is a compact subset of $U$, yielding

$$
\mathcal{H}^{Q-k}\left(\left\{p \in K \mid \tau(p)=t \text { and } d_{H} \tau(p) \text { is not surjective }\right\}\right)=0
$$

for a.e. $t \in \mathbb{R}^{k}$. If we take a countable family $\left\{K_{j}\right\}$ of compact subsets with union $U$ and we apply 19$\}$ to any of them, it follows that for a.e. $t \in \mathbb{R}^{k}$ we have

$$
\mathcal{H}^{Q-k}\left(\left\{p \in U \mid \tau(p)=t \text { and } d_{H} \tau(p) \text { is not surjective }\right\}\right)=0 .
$$

This proves the following corollary of Theorem 2.13 .

Corollary 2.15. Let $\tau: U \rightarrow \mathbb{R}^{k}$ be a $C^{1}$ map. Then $\tau \in C_{H}^{1}\left(U, \mathbb{R}^{k}\right)$ and 20 holds for a.e. $t \in \mathbb{R}^{k}$.

The next theorem is the main result of this paper.

Theorem 2.16 (Negligibility). Let $\Sigma \subset \Omega$ be a $C^{1}$ submanifold of codimension $k$. Then $\mathcal{H}^{Q-k}(C(\Sigma))=0$.

Proof. According to Definition 1.4, we fix a graded basis $\left(W_{1}, \ldots, W_{q}\right)$ and the associated graded coordinates given by $F: \mathbb{R}^{q} \rightarrow \mathbb{G}$. Let us fix a point $p \in \Sigma$. By definition of $C^{1}$ submanifold there exists an open neighbourhood $O_{p}$ of $p$, a map $u_{p} \in C^{1}\left(O_{p}, \mathbb{R}^{k}\right)$ and some integers $1 \leq j_{1}<\cdots<j_{k} \leq q$ such that for any $s \in O_{p}$ the vectors $\left(W_{j_{1}} u_{p}(s), \ldots, W_{j_{k}} u_{p}(s)\right)$ are linearly independent and $\Sigma \cap O_{p}=u_{p}^{-1}(0)$. Proposition 2.12 permits us to translate $p$ to the unit element $e \in \mathbb{G}$.

We define $O=l_{p^{-1}} O_{p}$ and $u: O \rightarrow \mathbb{R}^{k}$ as $u(s)=u_{p}\left(l_{p} s\right)$ for each $s \in O$. The left invariance of the vector fields $W_{j}$ gives $W_{j} u(s)=W_{j} u_{p}\left(l_{p} s\right)$ for every $s \in O$ and every $j=1, \ldots, q$. Thus for every $s \in O$ the vectors $\left(W_{j_{1}} u(s), \ldots, W_{j_{k}} u(s)\right)$ are linearly independent. We define the translated submanifold $\Sigma_{p}=l_{p^{-1}} \Sigma$, the open set $\tilde{O}=F^{-1}(O) \subset \mathbb{R}^{q}$ and $\tilde{\Sigma}=F^{-1}\left(\Sigma_{p}\right) \subset \mathbb{R}^{q}$, observing that $\tilde{u}^{-1}(0)=\tilde{\Sigma} \cap \tilde{O}$ where $\tilde{u}=u \circ F: \tilde{O} \rightarrow \mathbb{R}^{k}$ is $C^{1}$. We have

$$
\partial_{x_{j}} \tilde{u}(0)=d u \circ \partial_{x_{j}} F(0)=d u \circ W_{j}(e)=W_{j} u(e)
$$


for any $j=1, \ldots, q$. Then the vectors $\left(\partial_{x_{j_{1}}} \tilde{u}, \ldots, \partial_{x_{j_{k}}} \tilde{u}\right)$ are linearly independent in an open neighbourhood $O_{1} \subset \tilde{O}$ of the origin. Let us define the subspace

$$
\Pi=\left\{x \in \mathbb{R}^{q} \mid x_{j_{1}}=\cdots=x_{j_{k}}=0\right\} .
$$

By the implicit function theorem there exist an open subset $A \subset \Pi$ containing the origin and a $C^{1}$ map $\varphi: A \rightarrow \mathbb{R}^{k}$ such that $\tilde{u}(\xi, \varphi(\xi))=0$ for any $\xi \in A$. Our notation precisely means

$$
(\xi, \varphi(\xi))=\sum_{i \notin\left\{j_{1}, \ldots, j_{k}\right\}} \xi_{i} e_{i}+\sum_{i \in\left\{j_{1}, \ldots, j_{k}\right\}} \varphi^{i}(\xi) e_{j_{i}}
$$

where $\left(e_{j}\right)$ is the canonical basis of $\mathbb{R}^{q}$. The map $\Phi: A \rightarrow O_{1}$ is defined by $\Phi(\xi)=$ $(\xi, \varphi(\xi)) \in \tilde{\Sigma}$ for any $\xi \in A$. We introduce the $C^{1}$ map $\Psi: \mathbb{R}^{k} \times A \rightarrow \mathbb{G}$,

$$
\Psi(t, \xi)=\exp t_{1} W_{j_{1}} \cdot \exp t_{2} W_{j_{2}} \cdots \exp t_{k} W_{j_{k}} \cdot F(\Phi(\xi))
$$

for every $(t, \xi) \in \mathbb{R}^{k} \times A$. We have used the dot to denote the group operation. We have

$$
\partial_{t_{i}} \Psi(0)=W_{j_{i}}(e) \in T_{e} \mathbb{G} \quad \text { and } \quad \partial_{\xi_{j}} \Psi(0)=W_{j}(e)+\sum_{i=1}^{k} \varphi_{\xi_{j}}^{j_{i}}(0) W_{j_{i}}(e),
$$

for every $i=1, \ldots, k$ and every $j \notin\left\{j_{1}, \ldots, j_{k}\right\}$. It is easily seen that the vectors

$$
\left(\partial_{t_{1}} \Psi(0), \ldots, \partial_{t_{q}} \Psi(0)\right)
$$

are a basis of $T_{e} \mathbb{G}$, hence there exist $\varepsilon>0$ and open sets $A_{1} \subset A$ and $U \subset \mathbb{G}$ such that $\Psi\left((-\varepsilon, \varepsilon)^{k} \times A_{1}\right)=U, 0 \in A_{1}, e \in U$ and the function

$$
\Psi:(-\varepsilon, \varepsilon)^{k} \times A_{1} \rightarrow U
$$

is invertible. Let us consider the projection $\mathfrak{p}: \mathbb{R}^{q} \rightarrow \mathbb{R}^{k}$ defined by $\mathfrak{p}(x)=\left(x_{j_{1}}, \ldots, x_{j_{k}}\right)$ for any $x \in \mathbb{R}^{q}$ and define the $C^{1}$ map $\tau: U \rightarrow(-\varepsilon, \varepsilon)^{k}$ as $\tau(s)=\mathfrak{p}\left(\Psi^{-1}(s)\right)$ for any $s \in U$. Then Corollary 2.15 implies that for a.e. $t \in(-\varepsilon, \varepsilon)^{k}$ we have

$$
\mathcal{H}^{Q-k}\left(\left\{s \in U \mid \tau(s)=t \text { and } d_{H} \tau(s) \text { is not surjective }\right\}\right)=0 .
$$

As $\Psi$ is invertible it follows that $d \tau(s)$ is surjective for every $s \in U$, so the subset $S_{t}=$ $\tau^{-1}(t) \subset U$ is a $C^{1}$ submanifold for any $t$. We can apply Lemma 2.11 to obtain

$$
C\left(S_{t}\right)=\left\{s \in S_{t} \mid d_{H} \tau(s) \text { is not surjective }\right\} .
$$

In view of 21) we get $\mathcal{H}^{Q-k}\left(C\left(S_{t}\right)\right)=0$ for a.e. $t \in(-\varepsilon, \varepsilon)^{k}$. By definition of $\tau$ we know that

$$
\begin{aligned}
S_{t} & =\tau^{-1}(t)=\left\{s \in U \mid \Psi^{-1}(s) \in\{t\} \times A_{1}\right\} \\
& =\exp t_{1} W_{j_{1}} \cdot \exp t_{2} W_{j_{2}} \cdots \exp t_{k} W_{j_{k}} \cdot F\left(\Phi\left(A_{1}\right)\right) \\
& =l_{s_{t_{1}}}\left(l_{s_{t_{2}}}\left(\cdots\left(l_{s_{t_{k}}} F\left(\Phi\left(A_{1}\right)\right)\right)\right) \cdots\right),
\end{aligned}
$$


where $t=\left(t_{1}, \ldots, t_{k}\right)$ and $s_{t_{i}}=\exp t_{i} W_{j_{i}}$ for every $i=1, \ldots, k$. For $t=0 \in \mathbb{R}^{k}$ the previous equations yield $S_{0}=F\left(\Phi\left(A_{1}\right)\right) \subset \Sigma_{p}$, so

$$
S_{t}=l_{s_{t_{1}}}\left(l_{s_{t_{2}}}\left(\cdots\left(l_{s_{t_{k}}} S_{0}\right)\right) \cdots\right) .
$$

This formula and the $\mathcal{H}^{Q-1}$-negligibility of $C\left(S_{t}\right)$ for a.e. $t \in(-\varepsilon, \varepsilon)^{k}$ yield

$$
\begin{aligned}
0 & =\mathcal{H}^{Q-k}\left(C\left(S_{t}\right)\right)=\mathcal{H}^{Q-k}\left(C\left(l_{s_{t_{1}}}\left(l_{s_{t_{2}}}\left(\cdots\left(l_{s_{t_{k}}} S_{0}\right)\right) \cdots\right)\right)\right) \\
& =\mathcal{H}^{Q-k}\left(l_{s_{t_{k}}}\left(l_{s_{t_{k-1}}} \cdots\left(l_{s_{t_{1}}}\left(C\left(S_{0}\right)\right) \cdots\right)\right)\right)=\mathcal{H}^{Q-k}\left(C\left(S_{0}\right)\right) .
\end{aligned}
$$

In the third equality we have used Proposition 2.12 and in the fourth one we have used the fact that left translations are isometries with respect to any homogeneous distance.

Note that $S_{0}$ contains the unit element $e$ and it is an open subset of $\Sigma_{p}$. We know that $\Sigma_{p}=l_{p^{-1}} \Sigma$, hence defining $S_{p}=l_{p} S_{0}$ we see that $S_{p} \subset \Sigma$ is an open neighbourhood of $p$ in the topology of $\Sigma$. Thus, reasoning as before we obtain

$$
\mathcal{H}^{Q-k}\left(S_{p}\right)=\mathcal{H}^{Q-k}\left(C\left(l_{p} S_{0}\right)\right)=\mathcal{H}^{Q-k}\left(l_{p}\left(C\left(S_{0}\right)\right)\right)=\mathcal{H}^{Q-k}\left(C\left(S_{0}\right)\right)=0 .
$$

The arbitrary choice of $p$ implies that we can find a countable family of open subsets

$$
\left\{S_{p_{j}} \mid S_{p_{j}} \subset \Sigma, j \in \mathbb{N}\right\}
$$

such that $\Sigma=\bigcup_{j \in \mathbb{N}} S_{p_{j}}$ and $\mathcal{H}^{Q-k}\left(C\left(S_{p_{j}}\right)\right)=0$ for every $j \in \mathbb{N}$. Finally, the conclusion follows from the equality $C(\Sigma)=\bigcup_{j \in \mathbb{N}} C\left(S_{p_{j}}\right)$.

A first important consequence of the previous theorem occurs in codimension one, where we obtain the representation of the $(Q-1)$-dimensional spherical Hausdorff measure of $C^{1}$ hypersurfaces. To see this, we will use the notion of "metric factor" [48].

Definition 2.17 (Metric factor). Let $B_{1}$ be the open unit ball with respect to a fixed homogeneous distance $d$ of $\mathbb{G}$. Consider $v \in H^{1} \backslash\{0\}$ along with its orthogonal hyperplane $\tilde{\mathcal{L}}(v)$ in $\mathcal{G}$ and define $\mathcal{L}(v)=\exp \tilde{\mathcal{L}}(v) \subset \mathbb{G}$. Let $F: \mathbb{R}^{q} \rightarrow \mathbb{G}$ represent a system of graded coordinates and define

$$
\theta_{Q-1}^{g}(v)=\mathcal{H}_{|\cdot|}^{q-1}\left(F^{-1}\left(\mathcal{L}(v) \cap B_{1}\right)\right) .
$$

The map $v \mapsto \theta_{Q-1}^{g}(v)$ is called the metric factor of the homogeneous distance $d$ with respect to the direction $v$ and the graded metric $g$.

With a slight abuse of notation, it will be useful to define the metric factor also for horizontal vectors. We define

$$
\theta_{Q-1}^{g}(v)=\theta_{Q-1}^{g}(V)
$$

where $v \in H_{p} \mathbb{G}$ and $V \in H^{1} \backslash\{0\}$ is the unique vector field such that $V(p)=v$. Note that a more rigorous, but unnecessary formulation should consider the metric factor $\theta_{Q-1}^{g}$ as a function on the tangent bundle $T \mathbb{G}$ and which is constant along left invariant vector fields. 
Remark 2.18. As we have seen in Lemma 1.10 of [48], the above definition does not depend on the choice of graded coordinates. So the number $\theta_{Q-1}^{g}(v)$ depends only on the homogeneous distance $d$, the direction of $v$ and the graded metric $g$. By the symmetry of Euclidean balls, taking two of them $B_{r_{1}}^{|\cdot|}$ and $B_{r_{2}}^{|\cdot|}$ such that $B_{r_{1}}^{|\cdot|} \subset F^{-1}\left(B_{1}\right) \subset B_{r_{2}}^{|\cdot|}$, we notice that the intersection $F^{-1}(\mathcal{L}(v)) \cap B_{r_{i}}^{|\cdot|}$ for $i=1,2$ is independent of the direction $v$. As a result, for any $v \in H^{1} \backslash\{0\}$ we have

$$
\omega_{q-1} r_{1}^{q-1} \leq \theta_{Q-1}^{g}(v) \leq \omega_{q-1} r_{2}^{q-1}
$$

so $v \mapsto \theta_{Q-1}^{g}(v)$ is uniformly bounded from above and from below by positive constants which depend on the homogeneous distance and on the graded metric.

In Subsection 2.1 of [48] a class of stratified groups where the Carnot-Carathéodory distance yields a constant metric factor is singled out, namely, the class of $\mathcal{R}$-rotational groups, which encompasses Heisenberg groups and more general H-type groups. In the appendix of [29], Franchi, Serapioni and Serra Cassano have constructed a general homogeneous distance $d_{\infty}$ for every stratified group. Its explicit formula is stated in $\mathbb{R}^{q}$ with respect to graded coordinates:

$$
d_{\infty}(x, 0)=\max _{j=1, \ldots, l}\left\{\varepsilon_{j}\left|\left(x^{m_{j-1}+1}, \ldots, x^{m_{j}}\right)\right|^{1 / j}\right\}
$$

it has the left invariance property $d_{\infty}(x, y)=d_{\infty}\left(x^{-1} \cdot y, 0\right)$, and $\left.\varepsilon_{j} \in\right] 0,1[$ for every $j=$ $1, \ldots, q$ are suitable dimensional constants depending only on the group. The integers $m_{j}$ for $j=0, \ldots, \iota$ are introduced in Definition 1.4 and the dot between $x^{-1}$ and $y$ denotes the group operation in graded coordinates, according to the discussion following Definition 1.4. In the next proposition we prove that the metric factor with respect to this distance becomes a constant function of the horizontal direction $v$.

Proposition 2.19. Let $\theta_{Q-1}^{g}$ represent the metric factor with respect to the distance $d_{\infty}$. Then there exists $\alpha_{Q-1}>0$ such that $\theta_{Q-1}^{g}(\nu)=\alpha_{Q-1}$ for every $v \in H^{1} \backslash\{0\}$.

Proof. For any couple of horizontal vectors $\nu, \mu \in H \backslash\{0\}$ we can find an isometry $\tau: \mathcal{G} \rightarrow \mathcal{G}$ such that $\tau(v)=\mu$ and $\tau(\tilde{\mathcal{L}}(v))=\tilde{\mathcal{L}}(\mu)$, where $\tilde{\mathcal{L}}(v)$ and $\tilde{\mathcal{L}}(\mu)$ are the orthogonal spaces to $\nu$ and $\mu$, respectively. We view these orthogonal spaces in $\mathbb{G}$ by defining $\mathcal{L}(v)=\exp (\tilde{\mathcal{L}}(\nu))$ and $\mathcal{L}(\mu)=\exp (\tilde{\mathcal{L}}(\mu))$. Let $\left(W_{1}, \ldots, W_{q}\right)$ be a graded basis of $\mathcal{G}$, let $F: \mathbb{R}^{q} \rightarrow \mathbb{G}$ be the associated system of graded coordinates and define $I(x)=\sum_{j=1}^{q} x^{j} W_{j} \in \mathcal{G}$ for every $x \in \mathbb{R}^{q}$. By the expression of $d_{\infty}$ it is easy to see that the unit ball $\tilde{B}_{1} \subset \mathbb{R}^{q}$ with respect to $d_{\infty}$ is preserved under the family of isometries $\tilde{\tau}$, i.e. $\tilde{\tau}\left(\tilde{B}_{1}\right)=\tilde{B}_{1}$. Thus, taking into account that $F=\exp \circ I$ and that $\tilde{\tau}=I^{-1} \circ \tau \circ I$ : $\mathbb{R}^{q} \rightarrow \mathbb{R}^{q}$ is a Euclidean isometry, we have

$$
\begin{aligned}
F^{-1}(\mathcal{L}(\mu)) \cap \tilde{B}_{1} & =I^{-1}(\tilde{\mathcal{L}}(\mu)) \cap \tilde{B}_{1}=I^{-1}(\tau(\tilde{\mathcal{L}}(v))) \cap \tilde{B}_{1} \\
& =\tilde{\tau}\left(I^{-1}(\tilde{\mathcal{L}}(v)) \cap \tilde{\tau}^{-1}\left(\tilde{B}_{1}\right)\right)=\tilde{\tau}\left(I^{-1}(\tilde{\mathcal{L}}(v)) \cap \tilde{B}_{1}\right) \\
& =\tilde{\tau}\left(F^{-1}(\mathcal{L}(v)) \cap \tilde{B}_{1}\right),
\end{aligned}
$$


so by Definition 2.17 it follows that

$$
\begin{aligned}
\theta_{Q-1}^{g}(\mu) & =\mathcal{H}_{|\cdot|}^{q-1}\left(F^{-1}(\mathcal{L}(\mu)) \cap \tilde{B}_{1}\right)=\mathcal{H}_{|\cdot|}^{q-1}\left(\tilde{\tau}\left(F^{-1}(\mathcal{L}(\nu)) \cap \tilde{B}_{1}\right)\right) \\
& =\mathcal{H}_{|\cdot|}^{q-1}\left(F^{-1}(\mathcal{L}(v)) \cap \tilde{B}_{1}\right)=\theta_{Q-1}^{g}(v) .
\end{aligned}
$$

This concludes the proof.

In view of Theorem 2.16 in the case $k=1$, the statement of Theorem 2.3 in [48] holds for any $C^{1}$ submanifold of codimension one. As a consequence, the relation between the Riemannian measure $\sigma_{g}$ induced by the graded metric $g$ restricted to a $C^{1}$ submanifold $\Sigma$ of codimension one and $\mathcal{S}^{Q-1}\llcorner\Sigma$ is established on arbitrary stratified groups.

Theorem 2.20. Let $\Sigma$ be a $C^{1}$ submanifold of codimension one in $\Omega$. Then

$$
\begin{gathered}
\theta_{Q-1}^{g}\left(v_{H}\right) \mathcal{S}^{Q-1}\left\llcorner\Sigma=\left|v_{H}\right| \sigma_{g}\llcorner\Sigma,\right. \\
\mathcal{S}^{Q-1}\left\llcorner\Sigma=\frac{\left|v_{H}\right|}{\theta_{Q-1}^{g}\left(v_{H}\right)} \sigma_{g}\llcorner\Sigma,\right.
\end{gathered}
$$

where $\sigma_{g}$ is the measure induced by the graded metric g restricted to $\Sigma$.

\section{3. $\left(\mathbb{G}, \mathbb{R}^{k}\right)$-rectifiability and perimeter measure}

In this section we present some applications of Theorem 2.16 We start by introducing families of regular sets with respect to the geometry of stratified groups.

Definition 3.1 $\left(\left(\mathbb{G}, \mathbb{R}^{k}\right)\right.$-regular set). A subset $\Sigma \subset \Omega$ is a $\left(\mathbb{G}, \mathbb{R}^{k}\right)$-regular set if there exists a map $f \in C_{H}^{1}\left(\Omega, \mathbb{R}^{k}\right)$ such that $\Sigma=f^{-1}(0)$ and the P-differential

$$
d_{H} f(p): \mathbb{G} \rightarrow \mathbb{R}^{k}
$$

is a surjective G-linear map for any $p \in \Sigma$.

Definition 3.2 $\left(\left(\mathbb{G}, \mathbb{R}^{k}\right)\right.$-rectifiability). We say that $S \subset \Omega$ is $\left(\mathbb{G}, \mathbb{R}^{k}\right)$-rectifiable if there exists a sequence $\left\{\Sigma_{j}\right\}$ of $\left(\mathbb{G}, \mathbb{R}^{k}\right)$-regular sets such that

$$
\mathcal{H}^{Q-k}\left(S \backslash \bigcup_{j \in \mathbb{N}} \Sigma_{j}\right)=0 .
$$

The $\left(\mathbb{G}, \mathbb{R}^{k}\right)$-rectifiability turns out to have a "fractal nature" with respect to the Euclidean viewpoint, since $\left(\mathbb{G}, \mathbb{R}^{k}\right)$-regular sets may have Euclidean Hausdorff dimension strictly greater than their topological dimension, so they cannot be rectifiable in the Euclidean sense [41]. In the following definition we recall the classical notion of rectifiability, utilizing countable unions of $C^{1}$ surfaces, instead of Lipschitz images. 
Definition 3.3 (Rectifiability). We say that a subset $S \subset \mathbb{G}$ is rectifiable of codimension $k$ or simply $(q-k)$-rectifiable if there exists a sequence $\left\{\Sigma_{j}\right\}$ of $C^{1}$ surfaces of dimension $q-k$ such that

$$
\mathcal{H}_{|\cdot|}^{q-k}\left(S \backslash \bigcup_{j \in \mathbb{N}} \Sigma_{j}\right)=0 .
$$

Remark 3.4. Notice that $\left(\mathbb{G}, \mathbb{R}^{k}\right)$-rectifiability coincides with rectifiability when $\mathbb{G}=$ $\mathbb{R}^{q}$ and $q>k$. Thus the notion of $(\mathbb{G}, \mathbb{R})$-rectifiability allows us to state in a unified way the De Giorgi rectifiability theorem in stratified groups. By results of [19], [27], [29] we know that if $|\partial E|_{H}(\mathbb{G})<\infty$, where $\mathbb{G}$ is either a Euclidean space or a stratified group of step two, then the $H$-reduced boundary $\partial_{* H} E$ is $(\mathbb{G}, \mathbb{R})$-rectifiable. Presently, for stratified groups of step higher than two this result is an open issue.

In order to complete the picture, we briefly mention a further extension of the notion of $\left(\mathbb{G}, \mathbb{R}^{k}\right)$-rectifiability. In fact, we can replace $\mathbb{R}^{k}$ with another stratified group $\mathbb{M}$.

Definition $3.5((\mathbb{G}, \mathbb{M})$-regular set). A subset $\Sigma \subset \Omega$ is a $(\mathbb{G}, \mathbb{M})$-regular surface if there exist $f \in C_{H}^{1}(\Omega, \mathbb{M})$ such that $f^{-1}(e)=\Sigma$ and

$$
d_{H} f(p): \mathbb{G} \rightarrow \mathbb{M}
$$

is a surjective G-linear map for any $p \in \Sigma$.

It is apparent that the notion of $(\mathbb{G}, \mathbb{M})$-regularity in higher codimension leaves us a certain freedom in the choice of $\mathbb{M}$, but not all codomains are "good" to be considered. For instance, the family of $\left(\mathbb{H}^{n}, \mathbb{H}^{m}\right)$-regular sets is empty whenever $n>m$. This follows form the fact that there are no surjective G-linear maps from $\mathbb{H}^{n}$ onto $\mathbb{H}^{m}$, as can be checked by using the definition of G-linearity and the group operation in the Heisenberg group (see also Theorem 2.8 of [47]). As soon as we have a surjective G-linear map $L: \mathbb{G} \rightarrow \mathbb{M}$ a canonical example of a $(\mathbb{G}, \mathbb{M})$-regular set can be given by choosing the subgroup $N=L^{-1}(e) \subset \mathbb{G}$ which is $(\mathbb{G}, \mathbb{M})$-regular. In view of Proposition 1.12 of [47] the Hausdorff dimension of $N$ is $Q-P$, where $Q$ and $P$ are the Hausdorff dimensions of $\mathbb{G}$ and $\mathbb{M}$, respectively. These considerations lead to the following definition.

Definition 3.6 ( $(\mathbb{G}, \mathbb{M})$-rectifiability). We say that $S \subset \Omega$ is $(\mathbb{G}, \mathbb{M})$-rectifiable if there exists a sequence $\left\{\Sigma_{j}\right\}$ of $(\mathbb{G}, \mathbb{M})$-regular sets such that

$$
\mathcal{H}_{\rho}^{Q-P}\left(S \backslash \bigcup_{j \in \mathbb{N}} \Sigma_{j}\right)=0,
$$

where $\rho$ is the Carnot-Carathéodory distance on $\mathbb{G}$.

Theorem 3.7. Let $\Sigma \subset \Omega$ be a connected submanifold of class $C^{1}$ and codimension $k$. Then $\Sigma$ is $\left(\mathbb{G}, \mathbb{R}^{k}\right)$-rectifiable.

Proof. By Lemma 2.11 we see that $C(\Sigma)$ is a closed subset of $\Sigma$, so $\Sigma \backslash C(\Sigma)$ is a countable union of connected pieces that can be locally represented as level sets of $C^{1}$ maps with surjective P-differential. Theorem 2.16 implies $\mathcal{H}^{Q-k}(C(\Sigma))=0$, and hence the conclusion follows. 
Theorem 3.8. Every rectifiable set of codimension $k$ is $\left(\mathbb{G}, \mathbb{R}^{k}\right)$-rectifiable.

Proof. Let $S$ be a rectifiable set of codimension $k$ and let $\left\{\Sigma_{j} \mid j \in \mathbb{N}\right\}$ be a family of $C^{1}$ submanifolds of codimension $k$ such that $\mathcal{H}_{|\cdot|}^{q-k}\left(S \backslash \bigcup_{j \in \mathbb{N}} \Sigma_{j}\right)=0$. By Proposition 4.4 of [29], for every $\alpha>0$ and every $R>0$ there exists $C_{R}>0$ such that

$$
\mathcal{H}^{Q-q+\alpha}(E) \leq C_{R} \mathcal{H}_{|\cdot|}^{\alpha}(E)
$$

whenever $E \subset B_{R}$. Using the estimate (26) for $\alpha=q-k$ we obtain

$$
\mathcal{H}^{Q-k}\left(B_{R} \cap S \backslash \bigcup_{j \in \mathbb{N}} \Sigma_{j}\right) \leq \mathcal{H}_{|\cdot|}^{q-k}\left(B_{R} \cap S \backslash \bigcup_{j \in \mathbb{N}} \Sigma_{j}\right)=0
$$

for every $R>0$, so $\mathcal{H}^{Q-k}\left(S \backslash \bigcup_{j \in \mathbb{N}} \Sigma_{j}\right)=0$. By Theorem 3.7 we know that $\Sigma_{j}$ is $\left(\mathbb{G}, \mathbb{R}^{k}\right)$-rectifiable for any $j \in \mathbb{N}$. This concludes the proof.

Finally, we discuss an application of Theorem 2.16 related to a question raised by Danielli, Garofalo and Nhieu in [15]. They prove that there exist constants $c, C>0$ such that for every relatively compact open set $E$ with $C^{2}$ boundary the estimates

$$
c \mathcal{S}^{Q-1}(\partial E) \leq|\partial E|_{H}\left(\mathbb{H}^{n}\right) \leq C \mathcal{S}^{Q-1}(\partial E)
$$

hold, where $Q=2 n+2$ is the Hausdorff dimension of the Heisenberg group $\mathbb{H}^{n}$. They conjecture the validity of 277 for arbitrary stratified groups. Due to Theorem 2.5 of [49] (see also Theorem 3.5 of [28]) we have the formula

$$
|\partial E|_{H}=\theta_{Q-1}^{g}\left(v_{H}\right) \mathcal{S}^{Q-1}\llcorner\partial E,
$$

under the assumption that the characteristic set is $\mathcal{S}^{Q-1}$-negligible. Then Theorem 2.16 makes 28 true for any set $E$ with $C^{1}$ boundary. Thus, formula 28 immediately extends the validity of 277 to any open set with $C^{1}$ boundary contained in an arbitrary stratified group, yielding

$$
\underline{\theta}_{Q-1}^{g} \mathcal{S}^{Q-1}(\partial E \cap \Omega) \leq|\partial E|_{H}(\Omega) \leq \bar{\theta}_{Q-1}^{g} \mathcal{S}^{Q-1}(\partial E \cap \Omega),
$$

where $\Omega \subset \mathbb{G}$ is an arbitrary bounded open set and the constants $\underline{\theta}_{Q-1}^{g}, \bar{\theta}_{Q-1}^{g}$ are defined as follows:

$$
\underline{\theta}_{Q-1}^{g}=\inf _{v \in H^{1}} \theta_{Q-1}^{g}(v), \quad \bar{\theta}_{Q-1}^{g}=\sup _{v \in H^{1}} \theta_{Q-1}^{g}(v) .
$$

In Remark 2.18 we have shown that the function $v \mapsto \theta_{Q-1}^{g}(v)$ is bounded from above and from below by positive constants, therefore estimates $(29)$ are nontrivial.

As a last remark, we wish to show that the constants of 29] are actually optimal. To do this, we need the following definition. 
Definition 3.9. Let $p \in \mathbb{G}$ and let $v \in H^{1} \backslash\{0\}$. The vertical half spaces with respect to $v$ are defined as follows:

$$
\begin{aligned}
& S^{+}(v)=\exp (\{Y \in \mathcal{G} \mid\langle v, Y\rangle>0\}), \\
& S^{-}(v)=\exp (\{Y \in \mathcal{G} \mid\langle v, Y\rangle<0\}) .
\end{aligned}
$$

The vertical half spaces with respect to $v$ and centered at $p \in \mathbb{G}$ are defined as follows:

$$
l_{p}\left(S^{+}(v)\right)=S^{+}(p, v) \text { and } l_{p}\left(S^{-}(v)\right)=S^{-}(p, v) .
$$

Denote by $\mathcal{O}$ the family of nonempty bounded open sets of $\mathbb{G}$ and by $\mathcal{E}$ the family of open sets with $C^{1}$ boundary. We easily see that

$$
\begin{aligned}
\underline{\theta}_{Q-1}^{g} & \leq \inf \left\{\frac{|\partial E|_{H}(\Omega)}{\mathcal{S}^{Q-1}(\partial E \cap \Omega)} \mid \Omega \in \mathcal{O}, E \in \mathcal{E}\right\} \\
& \leq \inf \left\{\frac{\left|\partial S^{+}(v)\right|_{H}(\Omega)}{\mathcal{S}^{Q-1}\left(\partial S^{+}(\nu) \cap \Omega\right)} \mid \Omega \in \mathcal{O}, \nu \in H^{1} \backslash\{0\}\right\} \\
& =\inf \left\{\theta_{Q-1}^{g}(v) \mid v \in H^{1} \backslash\{0\}\right\}=\underline{\theta}_{Q-1}^{g},
\end{aligned}
$$

where the first equality of the last line follows from (28). We also have

$$
\begin{aligned}
\bar{\theta}_{Q-1}^{g} & \geq \sup \left\{\frac{|\partial E|_{H}(\Omega)}{\mathcal{S}^{Q-1}(\partial E \cap \Omega)} \mid \Omega \in \mathcal{O}, E \in \mathcal{E}\right\} \\
& \geq \sup \left\{\frac{\left|\partial S^{+}(\nu)\right|_{H}(\Omega)}{\mathcal{S}^{Q-1}\left(\partial S^{+}(v) \cap \Omega\right)} \mid \Omega \in \mathcal{O}, v \in H^{1} \backslash\{0\}\right\} \\
& =\sup \left\{\theta_{Q-1}^{g}(v) \mid v \in H^{1} \backslash\{0\}\right\}=\bar{\theta}_{Q-1}^{g} .
\end{aligned}
$$

This proves our claim.

Acknowledgments. The author wishes to thank the referees for their precious comments and observations.

\section{References}

[1] Ambrosio, L.: Some fine properties of sets of finite perimeter in Ahlfors regular metric measure spaces. Adv. Math. 159, 51-67 (2001) Zbl 1002.28004 MR 1823840

[2] Ambrosio, L.: Fine properties of sets of finite perimeter in doubling metric measure spaces. Set-Valued Anal. 10, 111-128 (2002) Zbl 1037.28002 MR 1926376

[3] Ambrosio, L., Fusco, N., Pallara, D.: Functions of Bounded Variation and Free Discontinuity Problems. Oxford Univ. Press (2000) Zbl 0957.49001 MR 1857292

[4] Ambrosio, L., Kirchheim, B.: Rectifiable sets in metric and Banach spaces. Math. Ann. 318, 527-555 (2000) Zbl 0966.28002 MR 1800768

[5] Ambrosio, L., Magnani, V.: Weak differentiability of BV functions on stratified groups. Math. Z. 245, 123-153 (2003) Zbl 1048.49030 MR 2023957

[6] Ambrosio, L., Serra Cassano, F., Vittone, D.: Intrinsic regular hypersurfaces in Heisenberg groups. Preprint (2005). 
[7] Ambrosio, L., Tilli, P.: Selected Topics on "Analysis in Metric Spaces". Scuola Norm. Sup., Pisa (2000) Zbl pre02043480 MR 2012736

[8] Balogh, Z. M.: Size of characteristic sets and functions with prescribed gradients. J. Reine Angew. Math. 564, 63-83 (2003) Zbl 1051.53024 MR 2021034

[9] Bellaïche, A.: The tangent space in sub-Riemannian geometry. In: Sub-Riemannian Geometry, Progr. Math. 144, A. Bellaïche and J. Risler (eds.), Birkhäuser, Basel, 1-78 (1996) Zbl 0862.53031 MR 1421822

[10] Bellaïche, A., Risler, J. J. (eds.): Sub-Riemannian Geometry. Progr. Math. 144, Birkhäuser, Basel (1996) Zbl 0848.00020 MR 1421821

[11] Capogna, L., Garofalo, N.: Boundary behavior of nonnegative solutions of subelliptic equations in NTA domains for Carnot-Carathéodory spaces. J. Fourier Anal. Appl. 4, 403-432 (1998) Zbl 0926.35043 MR 1658616

[12] Capogna, L., Garofalo, N.: Ahlfors regularity of the perimeter measure for minimally smooth hypersurfaces in Carnot-Carathéodory spaces. Preprint.

[13] Capogna, L., Garofalo, N., Nhieu, D. M.: Properties of harmonic measures in the Dirichlet problem for nilpotent Lie groups of Heisenberg type. Amer. J. Math. 124, 273-306 (2002) Zbl 0998.22001 MR 1890994

[14] Corwin, L., Greenleaf, F. P.: Representations of Nilpotent Lie Groups and their Applications. Part 1: Basic Theory and Examples. Cambridge Univ. Press (1990) Zbl 0704.22007 MR 1070979

[15] Danielli, D., Garofalo, N., Nhieu, D. M.: Trace inequalities for Carnot-Carathéodory spaces and applications. Ann. Scuola Norm. Sup. Pisa 27, 195-252 (1998) Zbl 0938.46036 MR 1664688

[16] Danielli, D., Garofalo, N., Nhieu, D. M.: Non-doubling Ahlfors measures, perimeter measures, and the characterization of the trace spaces of Sobolev functions in CarnotCarathéodory spaces. Mem. Amer. Math. Soc., to appear

[17] Danielli, D., Garofalo, N., Nhieu, D. M.: Minimal surfaces, area integral, sets of constant mean curvature and isoperimetry in Carnot groups. Preprint (2000)

[18] David, G., Semmes, S.: Fractured Fractals and Broken Dreams. Self-Similar Geometry through Metric and Measure. Oxford Univ. Press (1997) Zbl 0887.54001 MR 1616732

[19] De Giorgi, E.: Nuovi teoremi relativi alle misure $(r-1)$-dimensionali in uno spazio ad $r$ dimensioni. Ricerche Mat. 4, 95-113 (1955) Zbl 0066.29903 MR 0074499

[20] De Giorgi, E.: Problema di Plateau generale e funzionali geodetici. Atti Sem. Mat. Fis. Univ. Modena 43, 285-292 (1995) Zbl 0862.49028 MR 1366062

[21] Derridj, M.: Un problème aux limites pour une classe d'opérateurs du second ordre hypoelliptiques. Ann. Inst. Fourier (Grenoble) 21, no. 4, 99-148 (1971) Zbl 0215.45405 MR 0601055

[22] Derridj, M.: Sur un théorème de traces. Ann. Inst. Fourier (Grenoble) 22, no. 2, 73-83 (1972) Zbl 0231.46076 MR 0343011

[23] Federer, H.: Geometric Measure Theory. Springer (1969) Zbl 0176.00801 MR 0257325

[24] Fichera, G.: On a unified theory of boundary value problems for elliptic-parabolic equations of second order. In: Boundary Problems in Differential Equations, Univ. Wisconsin Press, Madison, 97-120 (1960) Zbl 0122.33504 MR 0111931

[25] Folland, G. B., Stein, E. M.: Hardy Spaces on Homogeneous Groups. Princeton Univ. Press (1982) Zbl 0508.42025 MR 0657581

[26] Franchi, B., Serapioni, R., Serra Cassano, F.: Meyers-Serrin type theorems and relaxation of variational integrals depending on vector fields. Houston J. Math. 22, 859-889 (1996) Zbl 0876.49014 MR 1437714 
[27] Franchi, B., Serapioni, R., Serra Cassano, F.: Rectifiability and perimeter in the Heisenberg group. Math. Ann. 321, 479-531 (2001) Zbl 1057.49032 MR 1871966

[28] Franchi, B., Serapioni, R., Serra Cassano, F.: Regular hypersurfaces, intrinsic perimeter and implicit function theorem in Carnot groups. Comm. Anal. Geom. 11, 909-944 (2003) Zbl pre02199004

[29] Franchi, B., Serapioni, R., Serra Cassano, F.: On the structure of finite perimeter sets in step 2 Carnot groups. J. Geom. Anal. 13, 421-466 (2003) Zbl 1064.49033

[30] Franchi, B., Serapioni, R., Serra Cassano, F.: Regular submanifolds, graphs and area formula in Heisenberg groups. Preprint (2004).

[31] Franchi, B., Wheeden, R. L.: Compensation couples and isoperimetric estimates for vector fields. Colloq. Math. 74, 9-27 (1997) Zbl 0915.46028 MR 1455453

[32] Garofalo, N., Nhieu, D. M.: Isoperimetric and Sobolev inequalities for Carnot-Carathéodory spaces and the existence of minimal surfaces. Comm. Pure Appl. Math. 49, 1081-1144 (1996) Zbl 0880.35032 MR 1404326

[33] Gromov, M.: Carnot-Carathéodory spaces seen from within. In: Sub-Riemannian Geometry, Progr. Math. 144, A. Bellaïche and J. Risler (eds.), Birkhäuser, Basel, 79-323 (1996) Zbl 0864.53025 MR 1421823

[34] Gromov, M.: Metric structures for Riemannian and non-Riemannian spaces. With appendices by M. Katz, P. Pansu, S. Semmes. Progr. Math. 152, J. Lafontaine and P. Pansu (eds.), Birkäuser, Boston (1999) Zbl 0953.53002 MR 1699320

[35] Hajłasz, P., Koskela, P.: Sobolev met Poincaré. Mem. Amer. Math. Soc. 145, no. 688 (2000) Zbl 0954.46022 MR 1683160

[36] Heinonen, J.: Lectures on Analysis on Metric Spaces. Springer, New York (2001) Zbl 0985.46008 MR 1800917

[37] Hochschild, G.: The Structure of Lie Groups. Holden-Day (1965) Zbl 0131.02702 MR 0207883

[38] Jerison, D.: The Dirichlet problem for the Kohn Laplacian on the Heisenberg group. Parts I and II. J. Funct. Anal. 43, 97-142 and 224-257 (1981) Zbl 0493.58022 MR 0639800

[39] Jerison, D.: Boundary regularity in the Dirichlet problem for $\square_{b}$ on CR manifolds. Comm. Pure Appl. Math. 36, 143-181 (1983) Zbl 0544.35069 MR 0690655

[40] Kirchheim, B., Magnani, V.: A counterexample to metric differentiability. Proc. Edinburgh Math. Soc. 46, 221-227 (2003) Zbl 1059.26007 MR 1961822

[41] Kirchheim, B., Serra Cassano, F.: Rectifiability and parametrization of intrinsic regular surfaces in the Heisenberg group. Ann. Scuola Norm. Sup. Pisa 3, 871-896 (2004) MR 2124590

[42] Kohn, J. J., Nirenberg, L.: Non-coercive boundary value problems. Comm. Pure Appl. Math. 18, 443-492 (1965) Zbl 0125.33302 MR 0181815

[43] Kohn, J. J., Nirenberg, L.: Degenerate elliptic-parabolic equations of second order. Comm. Pure Appl. Math. 20, 797-872 (1967) Zbl 0153.14503 MR 0234118

[44] Lanconelli, E., Uguzzoni, F.: On the Poisson kernel for the Kohn Laplacian. Rend. Mat. Appl. (7) 17, 659-677 (1997) Zbl 0908.35020 MR 1620876

[45] Leonardi, G. P., Rigot, S.: Isoperimetric sets on Carnot groups. Houston J. Math. 29, 609-637 (2003) Zbl 1039.49037 MR 2000099

[46] Magnani, V.: Differentiability and area formula on stratified Lie groups. Houston J. Math. 27, 297-323 (2001) Zbl 0983.22009 MR 1874099

[47] Magnani, V.: On a general coarea inequality and applications. Ann. Acad. Sci. Fenn. Math. 27, 121-140 (2002) Zbl 1064.49034 MR 1884354

[48] Magnani, V.: A blow-up theorem for regular hypersurfaces on nilpotent groups. Manuscripta Math. 110, 55-76 (2003) Zbl 1010.22010 MR 1951800 
[49] Magnani, V.: The coarea formula for real-valued Lipschitz maps on stratified groups. Math. Nachr. 278, 1689-1705 (2005) Zbl 1079.49030 MR 2176673

[50] Magnani, V.: Unrectifiability and rigidity in stratified groups. Arch. Math. (Basel) 83, 568576 (2004) Zbl 1062.22019 MR 2105335

[51] Margulis, G. A., Mostow, G. D.: Some remarks on the definition of tangent cones in a CarnotCarathéodory space. J. Anal. Math. 80, 299-317 (2000) Zbl 0971.58004 MR 1771529

[52] Mitchell, J.: On Carnot-Carathéodory metrics. J. Differential Geom. 21, 35-45 (1985) Zbl 0554.53023 MR 0806700

[53] Montgomery, R.: A Tour of Subriemannian Geometries, Their Geodesics and Applications. Amer. Math. Soc. (2002) Zbl 1044.53022 MR 1867362

[54] Monti, R.: Brunn-Minkowski and isoperimetric inequality in the Heisenberg group. Ann. Acad. Sci. Fenn. Math. 28, 99-109 (2003) Zbl pre01960606 MR 1976833

[55] Monti, R., Morbidelli, D.: Trace theorems for vector fields. Math. Z. 239, 747-776 (2002) Zbl 1030.46041 MR 1902060

[56] Monti, R., Morbidelli, D.: Regular domains in homogeneous groups. Trans. Amer. Math. Soc. 357, 2975-3011 (2005) Zbl 1067.43003 MR 2135732

[57] Monti, R., Serra Cassano, F.: Surface measures in some CC spaces. Calc. Var. Partial Differential Equations 13, 339-376 (2001) Zbl 1032.49045 MR 1865002

[58] Nagel, A., Stein, E. M., Wainger, S.: Balls and metrics defined by vector fields I: Basic properties. Acta Math. 155, 103-147 (1985) Zbl 0578.32044 MR 0793239

[59] Pansu, P.: Métriques de Carnot-Carathéodory et quasiisométries des espaces symétriques de rang un. Ann. of Math. 129, 1-60 (1989) Zbl 0678.53042 MR 0979599

[60] Pauls, S. D.: The large scale geometry of nilpotent Lie groups. Comm. Anal. Geom. 9, 951982 (2001) Zbl 1005.53033 MR 1883722

[61] Pauls, S. D.: A notion of rectifiability modelled on Carnot groups. Indiana Univ. Math. J. 53, 49-81 (2004) Zbl pre02109060 MR 2048183

[62] Pauls, S. D.: Minimal surfaces in the Heisenberg group. Geom. Dedicata 104, 201-231 (2004) Zbl 1054.49029 MR 2043961

[63] Rothschild, L. P., Stein, E. M.: Hypoelliptic differential operators and nilpotent groups. Acta Math. 137, 247-320 (1976) Zbl 0346.35030 MR 0436223

[64] Semmes, S.: Some Novel Types of Fractal Geometry. Oxford Univ. Press (2001) Zbl 0970.28001 MR 1815356

[65] Stein, E. M.: Harmonic Analysis. Princeton Univ. Press (1993) Zbl 0821.42001 MR 1232192

[66] Varopoulos, N. Th., Saloff-Coste, L., Coulhon, T.: Analysis and Geometry on Groups, Cambridge Univ. Press, Cambridge (1992) Zbl 0744.43006 MR 1218884

[67] Vodop'yanov, S. K.: $\mathcal{P}$-Differentiability on Carnot groups in different topologies and related topics. Proc. Anal. Geom., Sobolev Institute Press, Novosibirsk, 603-670 (2000) Zbl 0992.58005 MR 1847541 